NISSUNA UMANA INVESTIGAZIONE SI PUO DIMANDARE VERA SCIENZIA S'ESSA NON PASSA PER LE MATEMATICHE DIMOSTRAZIONI LEONARDO DA VINCI

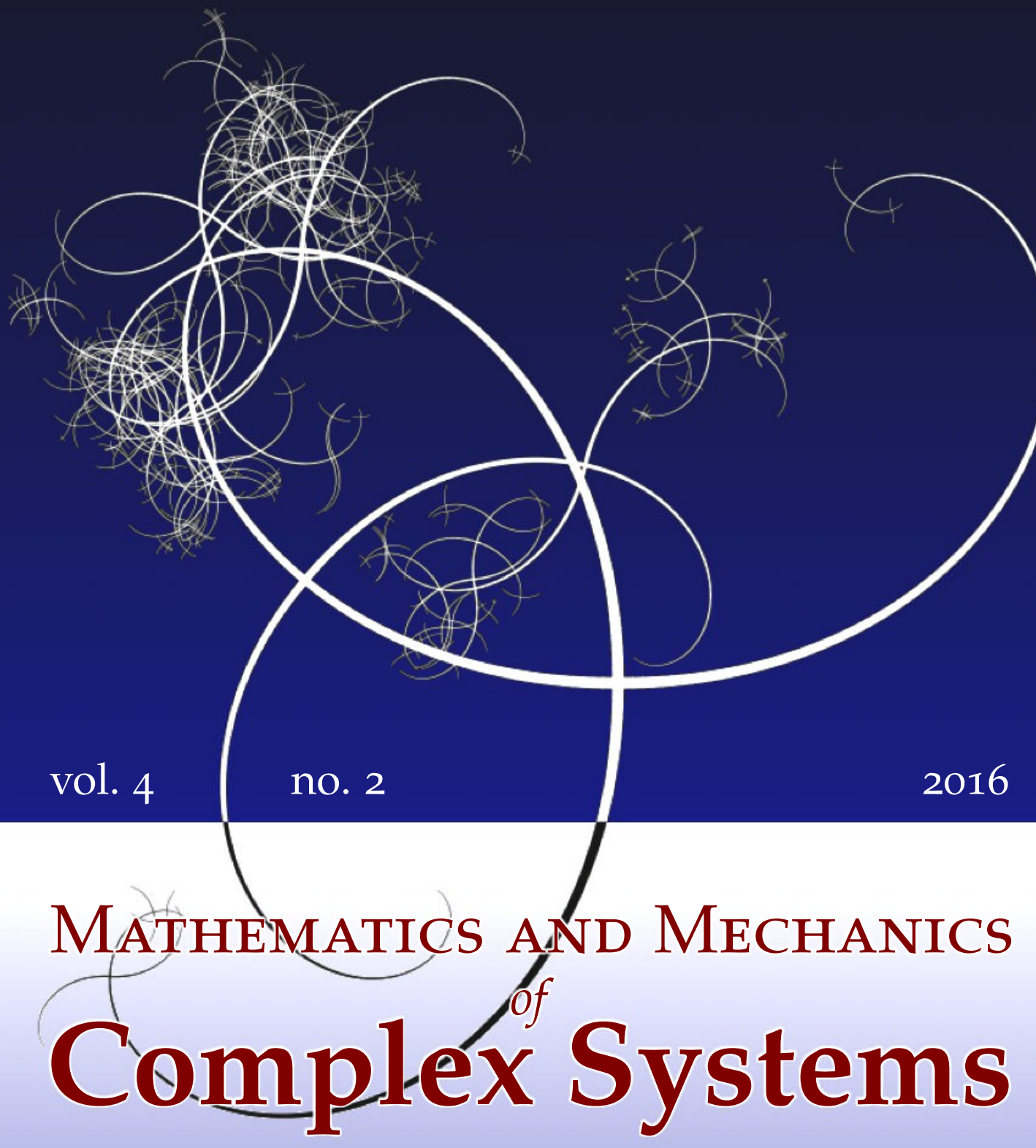

Nicolas Van Goethem

DISLOCATION-INDUCED LINEAR-ELASTIC STRAIN DYNAMICS BY A CAHN-HILLIARD-TYPE EQUATION 


\title{
DISLOCATION-INDUCED LINEAR-ELASTIC STRAIN DYNAMICS BY A CAHN-HILLIARD-TYPE EQUATION
}

\author{
NiCOLAS VAN GOETHEM
}

\begin{abstract}
In a single crystal containing dislocations, the elastic strain defined by a linear constitutive law from the stress tensor can be written as the sum of a symmetric gradient and a solenoidal tensor $\epsilon^{0}$, called the dislocation strain. This latter part of the elastic strain is related to dislocations since its incompatibility equals the curl of the contortion. The aim of this paper is to derive a time-evolution law for the internal thermodynamic variable $\epsilon^{0}$, arising from the second law of thermodynamics, and to discuss its mathematical setting. This encompasses a discussion on the functional space used and about the equation's well-posedness. A fourth-order time-dependent nonlinear PDE involving the incompatibility operator is found, which is similar in form to the Cahn-Hilliard equation, and represents in this respect a tensor generalization for solenoidal fields.
\end{abstract}

\section{Introduction and preliminary results}

Let $\Omega$ be a simply connected smooth and bounded subset of $\mathbb{R}^{3}$. Let $\mathcal{L}$ be a set of dislocation lines in $\Omega$ and the dislocation density $\Lambda_{\mathcal{L}} \in \mathcal{M}\left(\Omega, \mathbb{M}^{3}\right)$ be given by a Radon measure concentrated in $\mathcal{L}$. As soon as dislocations are present, the strain $\epsilon$ cannot be a symmetric gradient as the following crucial relation, called Kröner's formula, shows [Van Goethem 2016b]:

$$
\operatorname{inc} \epsilon=\operatorname{Curl} \kappa_{\mathcal{L}}, \quad \kappa_{\mathcal{L}}:=\Lambda_{\mathcal{L}}-\frac{\rrbracket_{2}}{2} \operatorname{tr} \Lambda_{\mathcal{L}},
$$

where $\mathbb{I}_{2}$ is the second-rank identity tensor and $\Lambda_{\mathcal{L}}$ the dislocation density tensor defined as $\Lambda_{\mathcal{L}}=\tau \otimes b \mathcal{H}^{1} \mathcal{L}_{\text {with }} \tau$ the tangent vector to the Lipschitz curve $\mathcal{L}$, $\mathcal{H}_{L \mathcal{L}}^{1}$ the one-dimensional Hausdorff measure concentrated in $\mathcal{L}$, and $b$ the Burgers vector, constant on the line. Moreover, inc is the incompatibility operator; i.e.,

$$
\text { inc } F:=\operatorname{Curl~Curl}^{t} F \text {, }
$$

\section{Communicated by Francesco dell'Isola.}

MSC2010: 35J48, 35J50, 35G31, 35K52, 35Q74.

Keywords: dislocations, linear elasticity, incompatibility, Cahn-Hilliard, evolution law, second principle. 
where the curl of a tensor is taken columnwise. This operator is at the heart of the present work since it will be shown to drive the time evolution of the dislocationinduced strain. Note that the evolution of the dislocations are given by the socalled contortion tensor $\kappa_{\mathcal{L}}$, which cannot be determined from the sole knowledge of its curl except in particular cases in which it is divergence-free, as for pure edge dislocations. For this reason, this work is not strictly speaking about the dynamics of dislocations.

Classically in linear elasticity, overall equilibrium reads $\operatorname{div} \mathbb{A} \epsilon=0$ in $\Omega$ with $\mathbb{A}$ the isotropic elasticity tensor. As a consequence, it is shown in [Van Goethem 2015] that there exist two fields of interest: the displacement $u$ and $F$, an auxiliary tensor that is solenoidal and symmetric. These fields satisfy Beltrami decomposition of the elastic strain, namely

$$
\epsilon=\nabla^{S} u+\operatorname{inc} F .
$$

In this paper, our aim is to derive an evolution law for the internal thermodynamic variable

$$
\epsilon^{0}:=\text { inc } F,
$$

which is called the dislocation-induced strain since it satisfies a regularized Kröner's relation inc $\epsilon^{0}=\operatorname{Curl} \kappa$, i.e., has a smoothed dislocation density (namely, the macroscopic contortion $\kappa$ ) in the right-hand side. Furthermore, $\epsilon^{0}$ satisfies a timedependent evolution that turns out to be sufficient for the global mechanical dissipation to be positive.

Specifically in this paper, we establish in a first step, study in a second step, and eventually discuss the nonlinear tensor-valued equation

$$
\alpha \partial_{t} \epsilon^{0}=\operatorname{inc}\left(-\mathbb{M} \text { inc } \epsilon^{0}-\mathcal{G}\left(\epsilon^{0}\right)\right) \quad \text { in } \Omega \times[0, T]
$$

with $\mathcal{G}$ a nonlinear potential, $\alpha>0$, and $\mathbb{M}$ a positive-definite and symmetric fourthorder material-dependent tensor. For simplicity, and for the sake of physical interpretation, we assume that $\mathcal{G}$ depends only on $e:=\operatorname{tr} \epsilon^{0}$, the trace of the dislocationinduced strain, which is shown to be directly related to and hence interpreted as a density of point defects. To achieve this goal, the mathematical nature of the incompatibility operator must be understood, and hence, a series of mathematical results must be recalled as preliminary steps.

Observe that evolution law (1-1) has a form similar to the Cahn-Hilliard equation but for a tensor-valued unknown $\epsilon^{0}$. Indeed, the Laplacian counterpart is precisely the incompatibility operator since $\operatorname{tr} \operatorname{inc} F=\Delta \operatorname{tr} F$, and hence, (1-1) appears as a tensor generalization for solenoidal tensor fields of the classical scalar Cahn-Hilliard equation. From a physical point of view, the scalar version of our equation is related to the dynamics of point defects, which are required for the creation and motion of dislocations and are related to the variation of matter density. 
Furthermore, $e$ obeys the scalar Cahn-Hilliard equation, though with nonstandard boundary conditions. A discussion about this equation, though derived by other means and with a different purpose, can be found in [Van Goethem 2014]. The purpose of this paper is to show that this equation is well-posed in an appropriate functional space; some of its important properties are given. Let us emphasize that particular care is given to justify the equation boundary conditions, which must be mathematically sound and at the same time have a physical interpretation.

The notion of internal variable of state. We consider $F$ a mathematical gauge field arising from Beltrami decomposition of symmetric tensors and without any particular physical meaning. However, its incompatibility, $\epsilon^{0}:=\operatorname{inc} F$, is the dislocation-induced strain since it is the only part of the elastic strain which appear in Kröner's formula. It is considered an internal variable of state (IVS) in the sense given here by G. Maugin [2015]: "Internal variables of state are introduced in thermomechanics in addition to the usual observable variables of state (e.g., deformation, temperature, electric, and magnetic fields). They are supposed to account in a more or less crude way for the complex internal microscopic processes that occur in the material and manifest themselves at a macroscopic scale in the form of dissipation".

Motivation. In our case, the observable variable of state (OVS) is the stress $\sigma$, from which the elastic strain $\epsilon$ is deduced by a constitutive law (hence, the latter is also an OVS). So far, $u$ and $F$ are vector and tensor fields involved in the decomposition of $\epsilon$. In some sense, $u$ is also observable, measurable, and controllable, depending on its boundary conditions and on the introduction of a reference configuration, which is an uncomfortable notion in infinitesimal elasticity. As a matter of fact, we prefer to let the identification $u$ be the displacement field as a convenient "vue de l'esprit".

The crucial point is that $\epsilon^{0}$ is an internal variable that is neither observable nor measurable or controllable, in the sense of physicists. Only its existence as a mathematical object and its effect in the form of dissipation is observed. Therefore, the aim of this paper is to show that it naturally obeys a PDE and thus becomes observable, measurable, and controllable in a mathematical sense. It should be emphasized that there exists no consensual procedure in the literature to determine the equation governing an IVS. Our plan is to derive such an equation in the simplest and most natural possible way, while not contradicting (at least) or, better, complying with (so far as possible) thermodynamics principles.

Structure of the work. The main part of this paper is about the derivation of the incompatibility-governed time-dependent model for the dislocation strain. To this end, considerations about the statics problem, and in particular about the choice 
of the boundary conditions and their physical meaning, are found in Sections 3, 3.1, and 3.2, respectively. The evolution law is then found in Section 4, whose mathematical properties, such as existence of solutions and energy bounds, are given in Section 5. In the preliminary Section 2, the functional spaces needed to mathematically handle the incompatibility operator are given. Several properties of tensor-valued fields with bounded incompatibility are also recalled, without proofs, to be found in a specifically dedicated paper [Amstutz and Van Goethem 2016]. A discussion is given in Section 6.

Notation and conventions. Let $E \in \mathbb{S}^{3}$ and $\beta \in \mathbb{M}^{3}$, where $\mathbb{M}^{3}$ denotes the space of square 3-matrices and $\mathbb{S}^{3}$ of symmetric 3-matrices. Note that superscript $t$ stands for the transpose of a tensor and superscript $S$ for the symmetric part of a tensor. The divergence and curl of a tensor $E$ are defined componentwise as (div $E)_{i}:=$ $\partial_{j} E_{i j}$ and $(\operatorname{Curl} E)_{i j}:=\epsilon_{j k l} \partial_{k} E_{i l}$, respectively. The incompatibility of a tensor $E$ is the symmetric tensor defined componentwise as

$$
(\operatorname{inc} E)_{i j}:=\left(\operatorname{Curl~Curl}^{t} E\right)_{i j}=\epsilon_{i k m} \epsilon_{j l n} \partial_{k} \partial_{l} E_{m n}=\left(\operatorname{Curl}^{t} \operatorname{Curl}^{t} E\right)_{i j} .
$$

Also, $(E \times N)_{i j}=-(N \times E)_{i j}=-\epsilon_{j k m} N_{k} E_{i m}$. Moreover, $\int_{\Omega} \operatorname{Curl} F \cdot E d x=$ $\int_{\Omega} F \cdot \operatorname{Curl} E d x$ and $\int_{\Omega}$ inc $F \cdot E d x=\int_{\Omega} F \cdot$ inc $E d x$ for smooth tensor-valued functions $E$ and $F$ with compact support in $\Omega$. It is a key part of this paper (see Section 2.1) to determine appropriate boundary conditions in order for this integration by parts to be valid for more general fields. We will also use the shorthand

$$
a \mid b:=\int_{\Omega} a \cdot b d x
$$

The following theorem is crucial for the developments of this work.

Theorem 1 (Beltrami decomposition [Maggiani et al. 2015]). Let $\Omega \subseteq \mathbb{R}^{3}$ be a simply connected domain with smooth boundary, let $p \in(1,+\infty)$ be a real number, and let $E \in L^{p}\left(\Omega, \mathbb{S}^{3}\right)$ be a symmetric tensor. Then there exist a vector field $u \in W^{1, p}\left(\Omega, \mathbb{R}^{3}\right)$ and a tensor $F \in L^{p}\left(\Omega, \mathbb{S}^{3}\right)$ with $\operatorname{Curl} F \in L^{p}\left(\Omega, \mathbb{S}^{3}\right)$, inc $F \in$ $L^{p}\left(\Omega, \mathbb{S}^{3}\right), \operatorname{div} F=0$ in $\Omega$, and $F N=0$ on $\partial \Omega$, where $N$ stands for the unit normal to $\partial \Omega$, satisfying

$$
E=\nabla^{S} u+\operatorname{inc} F
$$

Moreover, $u$ can be taken with vanishing trace on $\partial \Omega$, and such a pair $(u, F)$ is unique. 


\section{Preliminary results: functional spaces}

Define

$$
\begin{aligned}
H_{\text {curl }}\left(\Omega ; \mathbb{M}^{3}\right) & :=\left\{E \in L^{2}\left(\Omega ; \mathbb{M}^{3}\right): \operatorname{Curl} E \in L^{2}\left(\Omega, \mathbb{M}^{3}\right)\right\}, \\
\mathcal{H}(\Omega) & :=\left\{E \in H^{2}\left(\Omega, \mathbb{S}^{3}\right): \operatorname{div} E=0\right\}, \\
\mathcal{H}_{0}(\Omega) & :=\left\{E \in \mathcal{H}(\Omega): E=\operatorname{Curl}^{t} E \times N=0 \text { on } \partial \Omega\right\} .
\end{aligned}
$$

These spaces are naturally endowed with the Hilbertian structure of $H^{2}\left(\Omega, \mathbb{S}^{3}\right)$.

Some identities in the local basis. Let us consider the local orthonormal basis $\left(\tau^{A}, \tau^{B}, N\right)$ on $\partial \Omega$ (for detail on such bases and their extension in $\Omega$, see [Amstutz and Van Goethem 2016]). For a general symmetric tensor $T$, one has in this basis

$$
\begin{gathered}
T=\left(\begin{array}{lll}
T_{A A} & T_{A B} & T_{A N} \\
T_{B A} & T_{B B} & T_{B N} \\
T_{N A} & T_{N B} & T_{N N}
\end{array}\right), \quad T \times N=\left(\begin{array}{lll}
T_{A B} & -T_{A A} & 0 \\
T_{B B} & -T_{B A} & 0 \\
T_{N B} & -T_{N A} & 0
\end{array}\right), \\
(T \times N)^{t} \times N=\left(\begin{array}{ccc}
T_{B B} & -T_{A B} & 0 \\
-T_{A B} & T_{A A} & 0 \\
0 & 0 & 0
\end{array}\right) .
\end{gathered}
$$

By the same token,

and

$$
\begin{aligned}
& \left(T \times \tau^{A}\right)^{t} \times \tau^{A}=\left(\begin{array}{ccc}
0 & 0 & 0 \\
0 & T_{N N} & -T_{B N} \\
0 & -T_{N B} & T_{B B}
\end{array}\right), \\
& \left(T \times \tau^{B}\right)^{t} \times \tau^{B}=\left(\begin{array}{ccc}
T_{N N} & 0 & -T_{A N} \\
0 & 0 & 0 \\
-T_{N A} & 0 & T_{A A}
\end{array}\right),
\end{aligned}
$$

$$
\begin{aligned}
& \left(T \times \tau^{A}\right)^{t} \times \tau^{B}=\left(\begin{array}{ccc}
0 & 0 & 0 \\
-T_{N N} & 0 & T_{A N} \\
T_{N B} & 0 & -T_{A B}
\end{array}\right), \\
& \left(T \times \tau^{B}\right)^{t} \times \tau^{A}=\left(\begin{array}{ccc}
0 & -T_{N N} & T_{B N} \\
0 & 0 & 0 \\
0 & T_{N A} & -T_{B A}
\end{array}\right) .
\end{aligned}
$$

Similarly,

$$
\begin{aligned}
& (T \times N)^{t} \times \tau^{A}=\left(\begin{array}{ccc}
0 & T_{N B} & -T_{B B} \\
0 & -T_{N A} & T_{B A} \\
0 & 0 & 0
\end{array}\right), \\
& (T \times N)^{t} \times \tau^{B}=\left(\begin{array}{ccc}
-T_{N B} & 0 & T_{A B} \\
T_{N A} & 0 & -T_{A A} \\
0 & 0 & 0
\end{array}\right) .
\end{aligned}
$$


2.1. Green formula for the incompatibility operator. Let $V$ be a vector field defined on $\partial \Omega$, and let $\widetilde{V}$ be any extension of $V$ in $\Omega$ with appropriate regularity. The surface divergence of $V$ is defined on $\partial \Omega$ by

$$
\operatorname{div}_{S} V=\operatorname{div} \widetilde{V}-\left(\partial_{N} \widetilde{V}\right) \cdot N .
$$

The following result holding for smooth boundaries is sufficient for our purposes whereas, if the boundary had edges, an additional line-integral term must be added. Lemma 2 (surface divergence [Henrot and Pierre 2005]). If $V \in W^{1,1}\left(\partial \Omega, \mathbb{R}^{3}\right)$, then

$$
\int_{\partial \Omega} \operatorname{div}_{S} V d S(x)=\int_{\partial \Omega} \kappa V \cdot N d S(x) .
$$

Lemma 3 [Amstutz and Van Goethem 2016]. For all $U, V \in \mathcal{C}^{2}\left(\bar{\Omega}, \mathbb{M}^{3}\right)$,

$$
\int_{\Omega} U \cdot \operatorname{Curl} V d x=\int_{\Omega} \operatorname{Curl} U \cdot V d x+\int_{\partial \Omega}(U \times N) \cdot V d S(x) .
$$

Denote by $U^{S}=\left(U+U^{t}\right) / 2$ the symmetric part of a tensor $U$, and recall the definition of incompatibility (1-2). The following result is about integration by parts.

Lemma 4 [Amstutz and Van Goethem 2016]. Suppose that $T \in \mathcal{C}^{2}\left(\bar{\Omega}, \mathbb{S}^{3}\right)$ and $\eta \in H^{2}\left(\Omega, \mathbb{S}^{3}\right)$. Then

$$
\int_{\Omega} T \cdot \operatorname{inc} \eta d x=\int_{\Omega} \operatorname{inc} T \cdot \eta d x+\int_{\partial \Omega} \mathcal{T}_{1}(T) \cdot \eta d S(x)+\int_{\partial \Omega} \mathcal{T}_{0}(T) \cdot \partial_{N} \eta d S(x)
$$

with the trace operators defined as

$$
\begin{aligned}
& \mathcal{T}_{0}(T):=(T \times N)^{t} \times N, \\
& \mathcal{T}_{1}(T):=\left(\operatorname{Curl}(T \times N)^{t}\right)^{S}+\left(\left(\partial_{N}+\kappa\right) T \times N\right)^{t} \times N+\left(\operatorname{Curl}^{t} T \times N\right)^{S} .
\end{aligned}
$$

Remark 5. Only $\left(\partial_{N} \eta\right)_{T}$ matters in the rightmost integral of (2-7) since it can be equivalently rewritten as $\int_{\partial \Omega} \mathcal{T}_{0}(T) \cdot \mathcal{T}_{0}\left(\partial_{N} \eta\right) d S(x)$.

Remark 6. Let $\kappa^{R}$ be the two principal curvatures of $\partial \Omega$. It has been proved in [Amstutz and Van Goethem 2016] that ${ }^{1}$

$$
\operatorname{Curl}(T \times N)^{t}=-\sum_{R} \kappa^{R}\left(T \times \tau^{R}\right)^{t} \times \tau^{R}+\left(\operatorname{Curl}^{t} T \times N\right)^{t} .
$$

Taking an $\eta$ such that $\eta N=0=\partial_{N} \eta$ on $\partial \Omega$, then the boundary terms in (2-7) can be rewritten as

$$
\int_{\partial \Omega} \mathcal{T}_{1}(T) \cdot \eta d S(x)=\int_{\partial \Omega} \mathcal{T}_{1}(T)_{T} \cdot \eta_{T} d S(x)
$$

\footnotetext{
${ }^{1}$ The coefficient $\xi$ in [Amstutz and Van Goethem 2016] can be taken to be vanishing.
} 
Now, assuming that $\left(\operatorname{Curl}^{t} T \times N\right)^{S}=0$ and that $\mathcal{T}_{0}(T)=\mathcal{T}_{0}\left(\partial_{N} T\right)=0$ on $\partial \Omega$, taking into account (2-3), (2-9), and (2-10), the second Neumann boundary conditions, due to $(2-11)$, are

$$
\mathcal{T}_{1}(T)_{T}=-T_{N N} D N=0 \quad \text { on } \partial \Omega \text { where } D N=\left(\begin{array}{cc}
\kappa^{A} & 0 \\
0 & \kappa^{B}
\end{array}\right),
$$

with $T_{N N}=T N \cdot N$. In summary, we have the implication

$\left(\operatorname{Curl}^{t} T \times N\right)^{S}=\mathcal{T}_{0}(T)=\mathcal{T}_{0}\left(\partial_{N} T\right)=T N \cdot N=0 \quad$ and $\quad \eta N=0 \quad$ on $\partial \Omega$

$$
\Rightarrow \mathcal{T}_{1} \cdot \eta(T)=0 \text {. }
$$

Remark 7. The following alternative expression is also established in [Amstutz and Van Goethem 2016]:

$$
\begin{aligned}
\mathcal{T}_{1}(T)=-\sum_{R} \kappa^{R}\left(T \times \tau^{R}\right)^{t} \times \tau^{R}+\left(\left(-\partial_{N}+\kappa\right)\right. & T \times N)^{t} \times N \\
& -\sum_{R}\left(\partial_{R} T \times N\right)^{t} \times \tau^{R},
\end{aligned}
$$

where $\tau_{R}$ stands for the derivative along the $R$-th tangent vector $\tau^{R}$, for $R=A$ or $B$. Note that (2-14) is proved in [Amstutz and Van Goethem 2016, Lemma 3.19] by taking $\xi=0$ (since we consider smooth surfaces without umbilical points) and noting that each term of (2-14) is symmetric.

2.2. Basic properties. The following lemma is easy to prove from the properties of these functions.

Lemma 8. Every $E \in \mathcal{H}_{0}(\Omega)$ satisfies $\operatorname{div} \operatorname{Curl}^{t} E=0$ in $\Omega$ and $\operatorname{Curl}^{t} E \times N=$ $\partial_{N} E \times N=0$ on $\partial \Omega$. Moreover, inc $E|F=E|$ inc $F$ for every $E, F \in \mathcal{H}_{0}(\Omega)$.

Proof. The first statement comes easily from the solenoidal property of $E$. As for the second, compute componentwise (see [Amstutz and Van Goethem 2016] for detail)

$$
-\left[\operatorname{Curl}^{t} E \times N\right]_{m q}=\left(\left(\partial_{N} E \times N\right)^{t} \times N\right)_{m q}-\left(\left(\sum_{R} \tau^{R} \times \partial_{R} E\right)^{t} \times N\right)_{m q},
$$

where $\partial_{R}$ means the $R$-th tangential derivative, which here vanishes identically, proving the result. The last statement is a direct consequence of Lemma 4 and Remark 5, and taking into account the density of smooth functions in $\mathcal{H}_{0}(\Omega)$.

Lemma 9. Let $\Omega \subset \mathbb{R}^{3}$ be a bounded open set with boundary of class $\mathscr{C}^{1}$ and $F \in H_{\text {curl }}\left(\Omega ; \mathbb{M}^{3}\right)$ such that $F \times N=0$ on $\partial \Omega$. Then $(\operatorname{Curl} F) N=0$ on $\partial \Omega^{2}{ }^{2}$ Moreover, (inc $E) N=0$ on $\partial \Omega$ as soon as $E=\left(\partial_{N} E \times N\right)^{t} \times N=0$ on $\partial \Omega$.

\footnotetext{
${ }^{2}$ This expression is intended in a classical weak sense; see, e.g., [Van Goethem 2015].
} 
Proof. The first part is proven by taking an arbitrary $\varphi \in H^{2}\left(\Omega, \mathbb{R}^{3}\right)$ since by integration by parts and Lemma $3\langle(\operatorname{Curl} F) N, \varphi\rangle_{\partial \Omega}=\langle\operatorname{Curl} F, D \varphi\rangle=\langle F \times N, D \varphi\rangle_{\partial \Omega}=0$. The second part follows from the first part, the definition of incompatibility, and the identity $0=\operatorname{Curl}^{t} F \times N=0$ from Lemma 8 .

For a proof of the next lemma, see, e.g., [Kozono and Yanagisawa 2009; von Wahl 1992; Bolik and von Wahl 1997].

Lemma 10. Let $F \in H_{\text {curl }}\left(\Omega ; \mathbb{M}^{3}\right)$ such that $\operatorname{div} F=0$ in $\Omega$ and $F \times N=0$ on $\partial \Omega$. Then $F \in H^{1}\left(\Omega, \mathbb{M}^{3}\right)$, and

$$
\|\nabla F\|_{L^{2}(\Omega)} \leq C\|\operatorname{Curl} F\|_{L^{2}(\Omega)} .
$$

The next result follows without major difficulty from Lemma 10.

Lemma 11. For all $E \in \mathcal{H}_{0}(\Omega)$,

$$
\|E\|_{H^{2}(\Omega)} \leq C\left(\|E\|_{L^{2}(\Omega)}+\|\operatorname{Curl} E\|_{L^{2}(\Omega)}+\|\operatorname{inc} E\|_{L^{2}(\Omega)}\right) .
$$

The following theorem is nonclassical but also easy to prove.

Theorem 12 (Poincaré). Let $\partial \Omega_{0} \subset \partial \Omega$ be nonflat with $\mathcal{H}^{2}\left(\partial \Omega_{0}\right)>0$. There exists a constant $C>0$ such that, for each $u \in H^{1}\left(\Omega ; \mathbb{R}^{3}\right)$,

$$
\|u\|_{L^{2}(\Omega)} \leq C\left(\|\nabla u\|_{L^{2}(\Omega)}+\int_{\partial \Omega_{0}}|u \times N| d S\right) .
$$

Theorem 13 (coercivity [Amstutz and Van Goethem 2016]). Let $\Omega$ be a bounded and connected domain with $\mathcal{C}^{1}$ boundary, and let the nowhere-flat subset $\partial \Omega_{0} \subset \partial \Omega$ with $\mathcal{H}^{2}\left(\partial \Omega_{0}\right)>0$. There exists a constant $C>0$ such that, for each $E \in \mathcal{H}_{0}(\Omega)$,

$$
\|E\|_{H^{2}(\Omega)} \leq C \| \text { inc } E \|_{L^{2}(\Omega)} .
$$

\section{Kinematics with dislocations}

First, the complete equations deriving from conservation of momentum are provided. They turn out to be nonclassical since, in the presence of dislocations, an auxiliary tensor variable appears as well as a dislocation-induced force in the righthand side of the equilibrium equation. Second, we discuss the chosen boundary conditions from a mathematical and physical standpoint. Let us emphasize here that from now on the forces will be regularized so that all fields are assumed smooth. This will allow us to perform a thermodynamical study in a classical manner.

3.1. Governing PDEs. The elastic strain is given from the stress tensor $\sigma$ by $\epsilon:=$ $\mathbb{A}^{-1} \sigma$, where $\mathbb{A}$ is the assumed constant elasticity tensor, i.e., $\mathbb{A}=2 \mu \rrbracket_{4}+\lambda \rrbracket_{2} \otimes \mathbb{I}_{2}$, 
where $\mathbb{\square}_{4}$ and $\mathbb{\rrbracket}_{2}$ are the fourth- and second-rank identity tensors, respectively, ${ }^{3}$ with $\mu$ and $\lambda$ the Lamé coefficients. Conservation of linear momentum reads

$$
\left\{\begin{aligned}
\rho \frac{d v}{d t}-\operatorname{div} \mathbb{A} \epsilon & =f & & \text { in } \Omega, \\
\sigma N & =g & & \text { on } \partial \Omega,
\end{aligned}\right.
$$

where $\rho$ is the volumic mass and $v$ the velocity and with $f \in \mathscr{C}^{\infty}\left(\Omega, \mathbb{R}^{3}\right)$ and $g \in \mathscr{C}^{\infty}\left(\partial \Omega, \mathbb{R}^{3}\right)$ the volume and surface forces, respectively. By Beltrami decomposition (see Theorem 1), there exists a vector $u$ and a symmetric and solenoidal tensor $F$ such that

$$
\epsilon=\nabla^{S} u+\text { inc } F
$$

whereby, recalling the solenoidal property of

$$
\epsilon^{0}:=\text { inc } F \text {, }
$$

conservation of linear momentum is rewritten as

$$
\left\{\begin{aligned}
\rho \frac{d v}{d t}-\operatorname{div}\left(\mathbb{A} \nabla^{S} u\right) & =\mathcal{F}_{\mathcal{L}}:=f+\lambda \nabla \operatorname{tr}(\operatorname{inc} F) & & \text { in } \Omega, \\
\left(\mathbb{A} \nabla^{S} u\right) N & =g-\lambda \operatorname{tr}(\operatorname{inc} F) N & & \text { on } \partial \Omega .
\end{aligned}\right.
$$

Therefore, $u$ is called the generalized displacement field since it coincides with the displacement field in the absence of dislocations, i.e., for $\epsilon^{0}=\operatorname{inc} F=0$. Moreover, we set $v:=\frac{d u}{d t}$, the pointwise velocity.

The right-hand side of (3-3) depends on $F$, i.e., through $\operatorname{tr} \epsilon^{0}$, for which an equation must be found. To this end, we appeal to Kröner's relation, proved in [Van Goethem 2016b] and which reads inc $\epsilon=\operatorname{inc} \epsilon^{0}=\operatorname{Curl} \kappa_{\mathcal{L}}$, where the righthand side is a concentrated first-order distribution. However, in the present work, which deals with thermodynamic consideration, the right-hand side will be regularized by convolution with a certain divergence-free mollifier $\eta_{\rho}$ (this amounts to considering a tubular neighborhood of the line of some fixed radius $\rho$, which is a common practice in the dislocation literature). Thus, by (3-2),

$$
\left\{\begin{aligned}
\operatorname{incinc} F & =\mathbb{G}_{\rho}:=\operatorname{Curl} \kappa_{\mathcal{L}} \star \eta_{\rho} & & \text { in } \Omega, \\
F & =0 & & \text { on } \partial \Omega, \\
\left(\partial_{N} F \times N\right)^{t} \times N & =0 & & \text { on } \partial \Omega,
\end{aligned}\right.
$$

where the boundary conditions are chosen in such a way that (3-4) has a unique solution. Indeed, (3-3) and (3-4) are well-posed as discussed in [Van Goethem 2015]. Note that well-posedness in weak form is a direct consequence of coercivity as proved in Section 2. Other boundary conditions of Neumann or mixed type will

${ }^{3}$ Componentwise, $\left(\mathbb{\square}_{4}\right)_{i j k l}=\frac{1}{2}\left(\delta_{i k} \delta_{j l}+\delta_{i l} \delta_{j k}\right)$ and $\left(\mathbb{Q}_{2}\right)_{i j}=\delta_{i j}$. 
be discussed below. Furthermore, $\operatorname{div} \mathbb{G}_{\rho}=0$, and hence, there exists $\kappa$ called the regularized contortion such that

$$
\mathbb{G}_{\rho}=\operatorname{Curl} \kappa .
$$

Note that such a model is also discussed in [Van Goethem 2016a].

\subsection{Chosen boundary conditions.}

Boundary conditions for the gauge field F. The boundary conditions of (3-4) are of essential type (i.e., Dirichlet-like). Note that the first boundary condition on $F$ in (3-4) is required to satisfy the boundary conditions of Beltrami decomposition (3-2) of Theorem 1. Furthermore, it has been shown in Lemma 8 that the second boundary condition for $F$ implies that $\operatorname{Curl}^{t} F \times N=0$ on $\partial \Omega$, which in turn implies that $($ inc $F) N=0$ by Lemma 9 .

On the other hand, in order to determine the natural boundary conditions, a Green formula has been computed in Section 2.1. In particular, the formula shows that the second boundary condition on $F$ may be replaced by a condition on the tangential components of inc $F$. Specifically, the following equation with pure Neumann boundary conditions has a solution [Amstutz and Van Goethem 2016]:

$$
\left\{\begin{aligned}
\operatorname{inc}(\mathbb{M} \text { inc } F) & =\operatorname{Curl} \kappa & & \text { in } \Omega, \\
\mathcal{T}_{0}(\operatorname{inc} F) & =0 & & \text { on } \partial \Omega, \\
\mathcal{T}_{1}(\operatorname{inc} F) & =0 & & \text { on } \partial \Omega,
\end{aligned}\right.
$$

with $\mathbb{M}$ positive-definite and where $\mathcal{T}_{0}$ and $\mathcal{T}_{1}$ are the trace operators as defined in Lemma 4. Note that $\mathcal{T}_{0}(A):=(A \times N)^{t} \times N$ stands by (2-2) for the tangential components of tensor $A$ (in a different order). So we will write

$$
A_{T}:=\mathcal{T}_{0}(A),
$$

with subscript $T$ standing for tangential. To be precise, as a consequence of the Green formula, $\mathcal{T}_{0}$ (inc $\left.F\right)$ is the dual of $\left(\partial_{N} F\right)_{T}$ and $\mathcal{T}_{1}$ (inc $\left.F\right)$ is the dual of $F$. This and the above remark imply that either (inc $F$ ) $N$ or (inc $F)_{T}$ might be prescribed on the boundary but not both simultaneously.

Remark 14. Because (3-6) is given with Neumann boundary conditions, uniqueness might only hold in a quotient space. Specifically, term $\int_{\partial \Omega} \operatorname{Curl}^{t} F \times N d S(x)$ might not vanish in the right-hand side of the coercivity inequality. Hence, $F$ is fixed up to a gauge field $\widetilde{F}$ satisfying $\int_{\partial \Omega} \operatorname{Curl}^{t} \widetilde{F} \times N d S(x)=0$. This kind of detail is not of interest for the purpose of this work, but the curious reader may refer to [Amstutz and Van Goethem 2016].

With a view of the time-evolution model, we would like to justify the chosen boundary condition for $\epsilon^{0}:=\operatorname{inc} F$ as derived in the next section. To this end, 
we must find a set of mixed essential/natural boundary conditions on $F$ and its derivatives that imply $\mathcal{T}_{1}($ inc $F)=0$. First let us make a general remark. There are six unknowns for a fourth-order operator, and hence, twelve complementary conditions must be prescribed on the boundary (for the complete theory, we refer to [Agmon et al. 1964]). This is the case if the symmetric $F$ and $\left(\partial_{N} F\right)_{T}$ are set to zero, for instance, as for the homogeneous Dirichlet boundary condition. For the pure Neumann case, $\mathcal{T}_{0}$ provides three independent conditions and $\mathcal{T}_{1}$ six, whereby there exist three degrees of freedom unprescribed (whence the quotient space).

We assume that $\mathcal{T}_{0}(\operatorname{inc} F$ ) and only the normal components $F N$ are vanishing on the boundary. Then, referring to the Green-formula expression (2-7) and (2-9) with $T=\epsilon^{0}=$ inc $F$, we observe that the central term in the right-hand side of $\mathcal{T}_{1}\left(\epsilon^{0}\right)$ simplifies to $\mathcal{T}_{0}\left(\partial_{N} \epsilon^{0}\right)$. By (2-9) and (2-10), it remains to consider the term $\left(\left(\mathrm{Curl}^{t} \epsilon^{0}\right) \times N\right)^{S}$ and the first term on the right of (2-10). On the one hand, the term $\left(\operatorname{Curl}^{t} \epsilon^{0}\right) \times N$ is related to the dislocation rotation gradient since one recognizes $\mathrm{Curl}^{t} \epsilon$ as the Frank tensor, satisfying for a general strain $\epsilon$ (by Mitchell-CesaroVolterra decomposition and path integrations; see, e.g., [Maggiani et al. 2015])

$$
\nabla \omega=\operatorname{Curl}^{t} \epsilon,
$$

where $\omega$ is the rotation field. Thus, defining the divergence-free dislocation-induced rotation $\omega^{0}$ by means of $\nabla \omega^{0}:=\operatorname{Curl}^{t} \epsilon^{0}$, if we impose that $\omega^{0}$ be constant on $\partial \Omega$, then $\nabla \omega^{0} \times N=\operatorname{Curl}^{t} \epsilon^{0} \times N=0$ on $\partial \Omega$. This is interpreted as a condition of rigid dislocation-induced rotation of the crystal boundary.

Summarizing, by recalling (2-13), if one assumes $F N=0$ (three conditions), $\mathcal{T}_{0}($ inc $F)=\mathcal{T}_{0}\left(\partial_{N}\right.$ inc $\left.F\right)=0(3+3=6$ conditions $)$, and $\omega^{0}=$ constant on $\partial \Omega$ (two conditions), then the second Neumann boundary condition will be zero for a nonflat boundary if we also assume the additional condition $\left(\epsilon^{0}\right)_{N N}:=\epsilon^{0} N \cdot N=0$ (the twelfth and last condition). Note that, as a consequence of $\left(\epsilon^{0}\right)_{T}=\left(\epsilon^{0}\right)_{N N}=0$ on $\partial \Omega$, the trace of $\epsilon^{0}$ vanishes, i.e.,

Obviously,

$$
e:=\operatorname{tr} \epsilon^{0}=0 \quad \text { on } \partial \Omega
$$

$$
\partial_{R} e=\partial_{R} \operatorname{tr} \epsilon^{0}=0 \quad \text { for } R=A, B \text { on } \partial \Omega .
$$

As resulting from the above considerations, from now on in this work, the following equation for $F$ will be considered:

$\left\{\begin{aligned} \operatorname{inc}(\operatorname{inc} F) & =\operatorname{Curl} \kappa & & \text { in } \Omega, \\ F N & =0 & & \text { on } \partial \Omega, \\ \mathcal{T}_{0}(\operatorname{inc} F) & =0 & & \text { on } \partial \Omega, \\ (\operatorname{inc} F) N \cdot N & =0 & & \text { on } \partial \Omega, \\ \mathcal{T}_{0}\left(\partial_{N} \operatorname{inc} F\right) & =0 & & \text { on } \partial \Omega, \\ \operatorname{Curl}^{t}(\operatorname{inc} F) \times N & =0 & & \text { on } \partial \Omega .\end{aligned}\right.$


Furthermore, by elliptic regularity ${ }^{4}$ and the smoothness of $\kappa$, the fields $F$ and inc $F$ are also smooth.

Note that the third line of (3-10) implies that

$$
\epsilon^{0} \times N=-\epsilon_{A A}^{0} \tau_{A} \otimes \tau_{B}+\epsilon_{B B}^{0} \tau_{B} \otimes \tau_{A}+\epsilon_{A B}^{0}\left(\tau_{A} \otimes \tau_{A}-\tau_{B} \otimes \tau_{B}\right)=0 .
$$

Boundary condition for the dislocation strain $\epsilon^{0}$. We recall the following facts. The elastic strain can be written as $\epsilon=\mathbb{A}^{-1} \sigma=\nabla^{S} u+\epsilon^{0}$, where inc $\epsilon=$ inc $\epsilon^{0}=$ $\operatorname{Curl} \kappa$. The tensor $\epsilon^{0}=\operatorname{inc} F$ is called the dislocation strain since it is the only part of the elastic strain related to the dislocation density.

First note that the Neumann conditions $\mathcal{T}_{0}(\operatorname{inc} F)=0$ and $(\operatorname{inc} F) N \cdot N=0$ in (3-10) exactly mean that $\left(\epsilon_{0}\right)_{T}=0$ and $\left(\epsilon_{0}\right)_{N N}=0$, respectively. Thus, they naturally impose Dirichlet boundary conditions for $\epsilon^{0}$ though incomplete since the components $\left(\epsilon_{0} N\right) \cdot \tau^{R}$ remain unprescribed so far (with $\tau^{R}$ the $R$-th tangent vector to $\partial \Omega$ ). We also impose $\left(\operatorname{Curl}^{t} \epsilon^{0}\right) \times N=0$ in (3-10).

In order to chose the remaining boundary conditions for $\epsilon^{0}$, we will require that the following integration by parts be valid:

$$
\mathbb{M i n c} \epsilon^{0} \mid \text { inc } \epsilon^{0}=\operatorname{inc}\left(\mathbb{M} \text { inc } \epsilon^{0}\right) \mid \epsilon^{0} \text {. }
$$

As a consequence of (3-10), we already know that $\left(\epsilon^{0}\right)_{T}=\left(\partial_{N} \epsilon^{0}\right)_{T}=\left(\epsilon_{0}\right)_{N N}=0$ on $\partial \Omega$. Therefore, recalling that $\mathcal{T}_{0}\left(\mathbb{M}\right.$ inc $\left.\epsilon^{0}\right) \cdot \partial_{N} \epsilon^{0}=\left(\mathbb{M}\right.$ inc $\left.\epsilon^{0}\right) \cdot\left(\partial_{N} \epsilon^{0}\right)_{T}=0$ on $\partial \Omega$, in order for (3-12) to hold, it suffices to impose, by referring to Green formula (2-7) with $T=\mathbb{M}$ inc $\epsilon^{0}$, that the boundary integrand $\mathcal{T}_{1}\left(\mathbb{M}\right.$ inc $\left.\epsilon^{0}\right) \cdot \epsilon^{0}=0$ on $\partial \Omega$. Furthermore, by (2-14), only the $N R$-components (for $R=A, B)$ of $\mathcal{T}_{1}\left(\mathbb{M}\right.$ inc $\epsilon^{0}$ ) matter in this product since $\left(\epsilon^{0}\right)_{T}=\left(\epsilon_{0}\right)_{N N}=0$ on $\partial \Omega$. Then only the first and last terms of (2-14) are nonvanishing, and (2-14) is equivalently rewritten by virtue of $(2-3)-(2-5)$ as

$$
\kappa^{R^{*}}\left(\mathbb{M} \text { inc } \epsilon^{0}\right)_{R N}+\partial_{R}\left(\mathbb{M i n c} \epsilon^{0}\right)_{R^{*} R^{*}}-\partial_{R^{*}}\left(\mathbb{M} \text { inc } \epsilon^{0}\right)_{R R^{*},} \quad R=A, B,
$$

where $\tau_{R}$ stands for the derivative along the $R$-th tangent vector $\tau^{R}$ and $\kappa^{R}$ for the $R$-th principal curvature, for $R=A$ or $B$, and with $A^{*}=B$ and $B^{*}=A$. Note that, for a cylindrical boundary, the last two terms are recognized as a surface curl.

Let us remark that, by Lemma 9 , (inc $\left.\epsilon^{0}\right) N=0$ since $\left(\operatorname{Curl}^{t} \epsilon^{0}\right) \times N=0$ on $\partial \Omega$, and hence, if one assumes that $\mathbb{M}$ has the same symmetry as the isotropic elasticity tensor, then $\left(\mathbb{M} \text { inc } \epsilon^{0}\right)_{R N}=0$ and the first term in (3-13) vanishes. In this case, the boundary condition reduces to imposing a vanishing surface curl of $\mathbb{M} \epsilon^{0}$.

${ }^{4}$ The operator inc inc is $\Delta^{2}$ for symmetric solenoidal fields, and equation well-posedness is shown in [Van Goethem 2015]. See also Section 2. 
Summarizing, the following boundary conditions will be prescribed for $\epsilon^{0}$ :

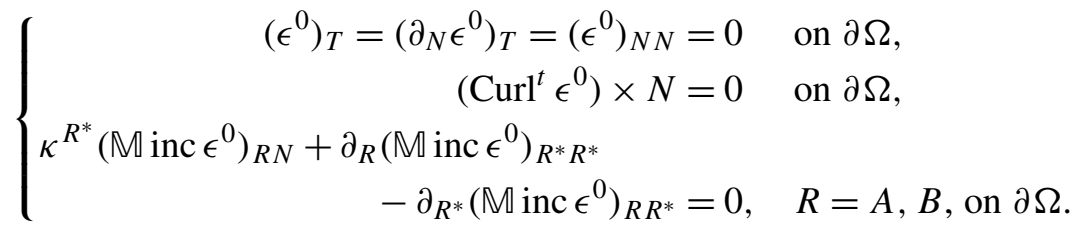

Let us recall that the Dirichlet conditions (i.e., the first two lines in (3-14)) follow from the chosen Neumann conditions for $F$, whereas the Neumann conditions are chosen so as to permit the integration by parts (3-12). As for their physical meaning, $\operatorname{Curl}^{t} \epsilon^{0}$ is the dislocation Frank tensor, i.e., the rotation gradient generated by the dislocations. Moreover, $\mathbb{M}$ inc $\epsilon^{0}=\mathbb{M} \operatorname{Curl} \kappa$ is a dislocation flux as related to the density of dislocation gradients and the crystal symmetries (given by the symmetries of tensor $\mathrm{M}$ ) and material properties. The Neumann condition is satisfied if for instance the dislocation density on the boundary is prescribed such that Curl $\kappa$ is purely tangential and constant on the boundary.

\section{Evolution law for the dislocation strain}

The aim of this section is to derive an evolution law for $\epsilon^{0}$ from the second principle of thermodynamics and by assuming that the evolution of the dislocation density (i.e., $\Lambda$ and hence $\kappa$ ) is known (by means of transport-reaction-diffusion $\mathrm{PDEs}^{5}$ ). To be precise, the model will be derived from a particular form of the global Clausius-Duhem inequality. Let us stress that the obtained evolution law is too simple to satisfy the principle in its full generality. In fact, our aim here is to derive a simple model based on the principle, study its mathematical wellposedness, and leave more elaborate models for future works. In this respect, our aim is also to show that the incompatibility operator naturally appears in the model as soon as high-order dislocation density terms are considered in the free energy. Note that evolution laws are often postulated from the statics equations, but this procedure is questionable since the resulting dynamics does not necessarily comply with thermodynamic principles.

\subsection{Model assumptions.}

Assumptions on the free energy. Let the Helmholtz free energy be given by

$$
\Psi:=\widehat{\Psi}(\epsilon, \kappa, \operatorname{Curl} \kappa)=\widehat{\Psi}_{e}(\epsilon)+\Psi_{\text {dislo }}\left(\epsilon^{0}, \kappa, \operatorname{Curl} \kappa\right),
$$

where a quadratic law in $\kappa$ and $\mathrm{Curl} \kappa$ is postulated:

$$
\Psi_{\text {dislo }}\left(\epsilon^{0}, \kappa, \operatorname{Curl} \kappa\right)=\frac{1}{2} \mathbb{N} \kappa \cdot \kappa+\frac{1}{2} \mathbb{M} \operatorname{Curl} \kappa \cdot \operatorname{Curl} \kappa+\psi_{\text {dislo }}\left(\epsilon^{0}\right)
$$

\footnotetext{
${ }^{5}$ For point defects, such a law was studied in [Van Goethem et al. 2008].
} 
with $\mathbb{M}$ and $\mathbb{N}$ positive-definite fourth-rank tensors. In this work, we will restrict ourselves to symmetric tensors $\mathbb{N}$ of the form

$$
\mathbb{N}=2 \beta \rrbracket_{4},
$$

where $\beta \geq 0$ is a constant scalar and $\left(\rrbracket_{4}\right)_{i j k l}=\frac{1}{2}\left(\delta_{i k} \delta_{j l}+\delta_{i l} \delta_{j k}\right)$. Note that $\mathbb{N}$ and hence $\beta$ have the dimensions of a force since $\kappa$, as $\Lambda$, has the dimensions of an inverse length while $\mathbb{M}$ has the dimensions of a force times a surface.

Let us emphasize that high-order dislocation models involving strain derivatives in the form of $\epsilon$ and its curl are not new; see, e.g., [Berdichevsky 2006].

Assumption of rigid dislocation-induced rotation. We assume that the dislocationinduced rotations are constant along $\partial \Omega$, that is, $\nabla \omega^{0} \times N=\operatorname{Curl}^{t} \epsilon^{0} \times N=0$. Nonetheless, variations of rotation may occur as induced by purely elastic loading since $\operatorname{Curl}^{t} \nabla^{S} u \times N \neq 0$.

Additional remark. The relation inc $\epsilon^{0}=\mathrm{Curl} \kappa$ yields

$$
\kappa=\operatorname{Curl}^{t} \epsilon^{0}+\nabla \varphi
$$

for some vector $\varphi$ satisfying, by the identity $\operatorname{div} \epsilon^{0}=0$,

$$
\mathcal{L}_{0,1}(\varphi)=\operatorname{div} \nabla^{S} \varphi=\operatorname{div} \kappa^{S},
$$

where we have chosen $\varphi=0$ on $\partial \Omega$. Note that this latter choice yields that $\operatorname{Curl}^{t} \epsilon^{0} \times N=0$ on $\partial \Omega$ implies that $\kappa \times N=0$ and hence $\operatorname{Curl} \kappa N=\operatorname{inc} \epsilon N=0$ on $\partial \Omega$, by Lemma 9 .

4.2. Thermodynamics considerations. The notions invoked in this section are classical in thermodynamics. References can be found in, e.g., [Lemaitre and Chaboche 1988; Ottosen and Ristinmaa 2005]. The idea is to derive an evolution law that would at least satisfy the second principle of thermodynamics globally in $\Omega$. The pointwise (otherwise termed local) isothermal Clausius-Duhem inequality reads

$$
\begin{aligned}
0 \leq D & =\sigma \cdot \nabla \dot{u}-\dot{\Psi} \\
& =\sigma \cdot(\dot{\epsilon}-\operatorname{inc} \dot{F})-\delta_{\epsilon} \Psi \cdot \dot{\epsilon}-\delta_{\kappa} \Psi \cdot \dot{\kappa}-\delta_{\operatorname{Curl} \kappa} \Psi \cdot \operatorname{Curl} \dot{\kappa}-\delta_{\epsilon} \Psi \cdot \dot{\epsilon}^{0} \\
& =-\sigma \cdot \operatorname{inc} \dot{F}+\dot{\epsilon}\left(\sigma-\delta_{\epsilon} \widehat{\Psi}_{e}\right)-\delta_{\kappa} \Psi_{\text {dislo }} \cdot \dot{\kappa}-\delta_{\operatorname{Curl} \kappa} \Psi_{\text {dislo }} \cdot \operatorname{Curl} \dot{\kappa}-\delta_{\epsilon^{0}} \psi_{\text {dislo }} \cdot \dot{\epsilon}^{0}
\end{aligned}
$$

(the $\cdot$ symbol stands for the time derivative, i.e., $\dot{\epsilon}:=\partial_{t} \epsilon(x, t)$, and $\nabla \dot{u}$ for the gradient of the velocity field). It is classically deduced that $\sigma=\delta_{\epsilon} \widehat{\Psi}_{e}$ and hence

$$
0 \leq D=-\sigma \cdot \operatorname{inc} \dot{F}-(\mathbb{N} \kappa \cdot \dot{\kappa}+\mathbb{M} \operatorname{Curl} \kappa \cdot \operatorname{Curl} \dot{\kappa})-\delta_{\epsilon^{0}} \psi_{\text {dislo }} \cdot \dot{\epsilon}^{0} .
$$

Introduce the global mechanical dissipation as

$$
\mathscr{D}:=\int_{\Omega} D d x
$$


The isothermal global form of the second law of thermodynamics (or the global Clausius-Duhem inequality) in $\Omega$ reads $^{6}$

$$
\mathscr{D} \geq 0 .
$$

Inequality (4-7) will allow us to derive the sought evolution equation for the dislocation strain. Recall the notation

$$
a \mid b:=\int_{\Omega} a \cdot b d x .
$$

By the symmetry of $\sigma$, Theorem 1 yields a unique $(\psi, \mathbb{S})$ satisfying $\sigma=\nabla^{S} \psi+$ inc $\mathbb{S}$ with $\psi=0, \mathbb{S}=0$, and $\mathrm{Curl}^{t} \mathbb{S} \times N=0$ on $\partial \Omega$ (the same remark as for (3-4) holds for $\mathbb{S}$ ). In particular, one has inc inc $\mathbb{S}=\operatorname{inc} \sigma$, where we remark that the dependence of $\mathbb{S}$ upon $\epsilon^{0}$ must not be linear. Furthermore,

$$
\sigma \cdot \operatorname{inc} \dot{F}=\nabla^{S} \psi \cdot \text { inc } \dot{F}+\text { inc } S \cdot \text { inc } \dot{F},
$$

which by integration by parts (justified by Lemma 8) yields

$$
\sigma \mid \text { inc } \dot{F}=\operatorname{inc} \mathbb{S} \mid \text { inc } \dot{F}=\mathbb{S} \mid \text { inc inc } \dot{F}=\mathbb{S} \mid \text { inc } \dot{\epsilon}=\mathbb{S} \mid \text { inc } \dot{\epsilon}^{0}=\text { inc } \mathbb{S} \mid \dot{\epsilon}^{0} \text {. }
$$

Moreover, by Beltrami decomposition again, the symmetric tensor $\delta_{\epsilon^{0}} \psi_{\text {dislo }}$ can be decomposed as

$$
\delta_{\epsilon^{0}} \psi_{\text {dislo }}=\nabla^{S} \eta+\text { inc } \mathbb{K}_{\epsilon^{0}}
$$

for some vector-valued $\eta$ (here taken with $\eta=0$ on $\partial \Omega$ ), where $\mathbb{K}_{\epsilon^{0}}$ is a symmetric divergence-free tensor whose dependence upon $\epsilon^{0}$ must not be linear too. Hence, recalling the solenoidal property of $\epsilon^{0}, \delta_{\epsilon^{0}} \psi_{\text {dislo }} \mid \dot{\epsilon}^{0}=$ inc $_{\mathbb{\epsilon}^{0}} \mid \dot{\epsilon}^{0}$. Thus, by (4-4), (4-6) and (4-7) can be rewritten as

$$
\begin{aligned}
0 \leq \mathscr{D}=-\operatorname{inc} \mathbb{S}_{\epsilon^{0}} \mid \dot{\epsilon}^{0}-\left(\mathbb { N } \left(\operatorname{Curl}^{t} \epsilon^{0}\right.\right. & +\nabla \varphi) \mid\left(\operatorname{Curl}^{t} \dot{\epsilon}^{0}+\nabla \dot{\varphi}\right) \\
& \left.+\mathbb{M} \text { inc } \epsilon^{0}\left|\operatorname{inc} \dot{\epsilon}^{0}+\operatorname{inc} \mathbb{K}_{\epsilon^{0}}\right| \dot{\epsilon}^{0}\right),
\end{aligned}
$$

where the dependence of $S$ upon $\epsilon^{0}$ has been emphasized by the subscript.

Let us now consider the second term of the right-hand side. By the symmetry property of $\mathbb{N}$ and since $\varphi=\dot{\varphi}=0$ on the boundary,

$$
\mathbb{N} \operatorname{Curl}^{t} \epsilon^{0}\left|\left(\operatorname{Curl}^{t} \dot{\epsilon}^{0}+\nabla \dot{\varphi}\right)=\mathbb{N} \operatorname{Curl}^{t} \epsilon^{0}\right| \operatorname{Curl} \dot{\epsilon}^{0}-\operatorname{div}\left(\mathbb{N} \operatorname{Curl}^{t} \epsilon^{0}\right) \mid \dot{\varphi} .
$$

Obviously, $\operatorname{div} \operatorname{Curl}^{t} \epsilon^{0}=\operatorname{div} \operatorname{Curl} \epsilon^{0}=0$, and hence, integrating $\mathbb{N} \operatorname{Curl}^{t} \epsilon^{0} \mid \operatorname{Curl} \dot{\epsilon}^{0}$ by parts by recalling (4-3) and Lemma 3 allows one to rewrite (4-12) as

$$
(4-12)=\beta \text { inc } \epsilon^{0}\left|\dot{\epsilon}^{0}+\beta \operatorname{Curl} \epsilon^{0}\right| \operatorname{Curl} \dot{\epsilon}^{0}+2 \beta \int_{\partial \Omega}\left(\operatorname{Curl}^{t} \epsilon^{0}\right)^{S} \times N \cdot \dot{\epsilon}^{0} d S,
$$

${ }^{6}$ The global form expressed in full generality would require a positive integral in any timedependent control volume in $\Omega$. 
where the integrand in the boundary term can be rewritten as $-\left(\operatorname{Curl}^{t} \epsilon^{0}\right)^{S} \cdot\left(\dot{\epsilon}^{0} \times N\right)$ and hence vanishes by (3-11). Now, by definition of the Frank tensor (3-7),

$$
\beta \operatorname{Curl} \epsilon^{0}\left|\operatorname{Curl} \dot{\epsilon}^{0}=\beta \operatorname{Curl}^{t} \epsilon^{0}\right| \operatorname{Curl}^{t} \dot{\epsilon}^{0}=\beta \nabla \omega^{0} \mid \nabla \dot{\omega}^{0} .
$$

Therefore, (4-13) can be rewritten as

$$
\beta \text { inc } \epsilon^{0}\left|\dot{\epsilon}^{0}+\beta \nabla \omega^{0}\right| \nabla \dot{\omega}^{0} .
$$

From the right-hand side of (4-11) and by $\operatorname{div} \operatorname{Curl}^{t} \epsilon^{0}=\operatorname{div} \operatorname{Curl} \epsilon^{0}=0$, one is left with

$$
\mathbb{N} \nabla \varphi\left|\left(\operatorname{Curl}^{t} \dot{\epsilon}^{0}+\nabla \dot{\varphi}\right)=\mathbb{N} \nabla^{S} \varphi\right| \nabla^{S} \dot{\varphi}=2 \beta \nabla^{S} \varphi \mid \nabla^{S} \dot{\varphi}
$$

with $\varphi$ the unique solution to (4-5). ${ }^{7}$

Summarizing, (4-11) is rewritten as

$$
\begin{aligned}
0 \leq \mathscr{D} & =-\beta \nabla \omega^{0}\left|\nabla \dot{\omega}^{0}-2 \beta \nabla^{S} \varphi\right| \nabla^{S} \dot{\varphi}-\beta \operatorname{inc} \epsilon^{0}\left|\dot{\epsilon}^{0}-\operatorname{inc}\left(\mathbb{M} \operatorname{inc} \epsilon^{0}+\mathbb{H}_{\epsilon^{0}}\right)\right| \dot{\epsilon}^{0} \\
& =-\frac{d}{d t} \mathscr{E}_{\beta}\left(\epsilon^{0}, \kappa\right)-\operatorname{inc}\left(\mathbb{M i n c} \epsilon^{0}+\beta \epsilon^{0}+\mathbb{H}_{\epsilon^{0}}\right) \mid \dot{\epsilon}^{0}
\end{aligned}
$$

where the nonlinear term with respect to $\epsilon^{0}$ is the symmetric and solenoidal tensor

$$
\mathbb{W}_{\epsilon^{0}}:=\mathbb{K}_{\epsilon^{0}}+\mathbb{S}_{\epsilon^{0}}
$$

and with a stored quadratic dislocation energy $\mathscr{E}_{\beta}$ defined as

$$
\mathscr{E}_{\beta}\left(\epsilon^{0}, \kappa\right):=\frac{\beta}{2}\left(\nabla \omega^{0}\left|\nabla \omega^{0}+2 \nabla^{S} \varphi\right| \nabla^{S} \varphi\right) .
$$

Let us remark that, if the free energy is independent of $\kappa$, that is, if $\beta=0$, then $\mathscr{E}_{\beta}=0$ and (4-16) immediately yields

$$
-\operatorname{inc}\left(\mathbb{M i n c} \epsilon^{0}+\beta \epsilon^{0}+\mathbb{W}_{\epsilon^{0}}\right) \mid \dot{\epsilon}^{0}=\mathscr{D} \geq 0 .
$$

4.3. Time-evolution of the dislocation strain. Let us now consider a certain time scale, which is lower than that of dissipative phenomena associated with the evolution of dislocations (the law for $\kappa$ ) but high enough not to invalidate the hypothesis of local state [Lemaitre and Chaboche 1988]. We will consider a thought experiment with a certain number of pure edge dislocations in such a way that $\varphi=0$, whereas the norm of $\nabla \omega^{0}$ can reach arbitrarily high values. Thus, one can render $-\frac{d}{d t} \mathscr{E}(\epsilon, \kappa)$ arbitrarily negative, and in order for the global dissipation $\mathscr{D}$ to remain positive in (4-16), the term inc $\left(\mathbb{M}\right.$ inc $\left.\epsilon^{0}+\beta \epsilon^{0}+\mathbb{W}_{\epsilon^{0}}\right) \mid \dot{\epsilon}^{0}$ must be nonpositive. For this reason, the following evolution law for $\epsilon^{0}$ is postulated:

$$
0=\alpha \dot{\epsilon}^{0}(t)+\operatorname{inc}\left(\mathbb{M} \text { inc } \epsilon^{0}(t)+\mathcal{G}\left(\epsilon^{0}(t)\right)\right)
$$

${ }^{7}$ Thus, it linearly depends on $\operatorname{div} \kappa^{S}$. 
for some material-dependent coefficient $\alpha \geq 0$ and with the solenoidal tensor-valued nonlinear term

$$
\mathscr{G}\left(\epsilon^{0}\right):=\mathbb{H}_{\epsilon^{0}}+\beta \epsilon^{0}=\mathcal{G}\left(\epsilon^{0}\right)-\mathbb{L},
$$

where $\mathbb{L}$ stands for a symmetric (not necessarily divergence-free) tensor independent of $\epsilon^{0}$. We introduce the generalized dislocation force as the symmetric and solenoidal tensor

$$
\mathbb{G}:=\text { inc } \mathbb{L} \text {. }
$$

Moreover, the boundary conditions (4-19) and the initial condition $\epsilon^{0}(0)=\epsilon_{0}^{0}$ at $t=0$ are prescribed.

Specifically, the sought time-dependent boundary-value problem for the dislocation strain reads, by recalling (3-14),

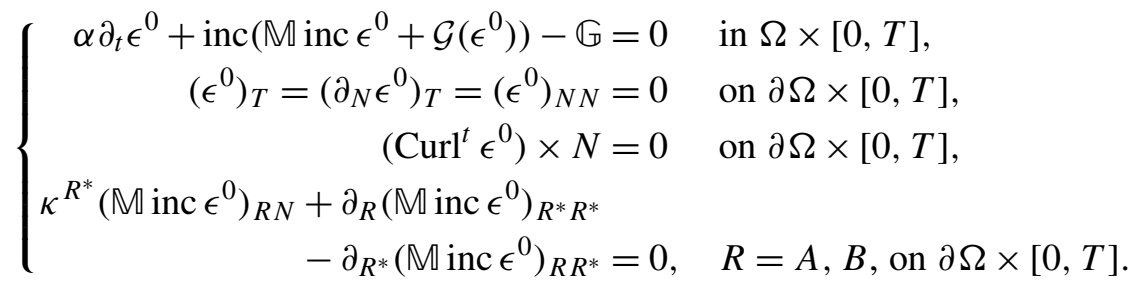

Furthermore, the following energy relation also holds:

$$
\frac{d}{d t} \mathscr{E}_{\beta}(\epsilon, \kappa) \leq \alpha\left|\dot{\epsilon}^{0}\right|^{2}
$$

In particular, the energy $\mathscr{E}_{\beta}$ decreases in time as soon as the dislocation strain is stationary.

\section{Well-posedness of the evolution}

5.1. Weak forms. Recall first the notation $a \mid b=(a, b)_{2}$, where the right-hand side stands for the scalar product in $L^{2}$ (of scalars, vectors, tensors, etc.). The weak form associated with (4-19) reads: for all $t \in[0, T]$, find $E(t) \in \mathcal{H}_{0}(\Omega)$ such that

$\alpha \frac{d E}{d t}(t) \mid F+\mathbb{M}$ inc $E(t)|\operatorname{inc} F+\mathcal{G}(E(t))| \operatorname{inc} F-\mathbb{G} \mid F=0$

$$
\text { for all } F \in \mathcal{H}_{0}(\Omega) \text {, }
$$

where $\mathbb{M}$ is a fourth-rank symmetric and positive-definite tensor, $\mathcal{G}$ is a symmetric tensor-valued nonlinear term (not necessarily divergence-free), $\mathbb{G}$ represents a tensor-valued generalized force, and $\alpha>0$, and such that

$$
E(0)=E_{0} \in L^{2}\left(\Omega ; \mathbb{S}^{3}\right) .
$$

\footnotetext{
${ }^{8}$ The dot and the partial time derivative symbols are equivalent. Recall that, in linearized elasticity, material and partial time derivatives do coincide.
} 
By integration by parts, and recalling Lemma 3, (5-1) can be rewritten as: find $E \in \mathcal{H}_{0}(\Omega)$ such that

$$
\begin{array}{r}
\alpha \frac{d E}{d t} \mid F+\mathbb{M} \text { inc } E|\operatorname{inc} F+\operatorname{Curl} \mathcal{G}(E)| \operatorname{Curl}^{t} F-\mathbb{G} \mid F=0 \\
\text { for all } F \in \mathcal{H}_{0}(\Omega) .
\end{array}
$$

The bilinear form associated with the linear part of the PDE reads

$$
a(E, F)=\mathbb{M} \text { inc } E \mid \text { inc } F \text {. }
$$

Its coercivity in $H^{2}(\Omega)$ is an immediate consequence of Theorem 13 .

Note that in the case of the dislocation model of Section 4.3, $\mathcal{G}\left(\epsilon^{0}\right)=\mathbb{H}_{\epsilon^{0}}+\beta \epsilon^{0}$. Recalling (4-18) and assuming for simplicity that $\mathbb{U}_{\epsilon^{0}}=\mathbb{U}(x)$ is independent of $\epsilon^{0}$, the weak form associated to this linear model can be written as: find $E \in \mathcal{H}_{0}(\Omega)$ such that

$$
\alpha \frac{d E}{d t} \mid F+(\mathbb{M} \text { inc } E+\beta E) \mid \text { inc } F-\widetilde{\mathbb{G}} \mid F=0 \quad \text { for all } F \in \mathcal{H}_{0}(\Omega)
$$

with $\widetilde{\mathbb{G}}:=\mathbb{G}+$ inc $\mathbb{T}$. In this case, the equation is a linearization of the general Cahn-Hilliard system.

Now, if $\mathcal{G}$ is assumed to be an objective tensor, it can be written in terms of its invariants, the first of which is the trace of $E$.

Assumption on the nonlinearity. The nonlinear term is assumed to be written as a polynomial in the trace of $E$ plus an affine term in $E$.

Assumption 15 (nonlinear term). Let $E \in \mathbb{S}^{3}$. It is assumed that

$$
\mathcal{G}(E)=\beta E-\frac{1}{3} \varphi(\operatorname{tr} E) \rrbracket_{2}
$$

with $\beta>0$ a constant scalar and $\varphi$ a scalar-valued polynomial defined as

$$
\varphi(v)=\sum_{i=1}^{2 p-1} \rho_{i} v^{i}, \quad p \geq 2,
$$

where $\rho_{2 p-1}>0$. In particular, $\mathcal{G}(E)$ is a symmetric second-rank tensor.

Remark 16. The divergence of the nonlinear term in (5-5) must not be zero since $\operatorname{div} \mathcal{G}(E)=-\frac{1}{3} \varphi^{\prime}(e) \nabla e \neq 0$ unless $\varphi$ is trivially independent of $e$. However, referring to the dislocation model of Section 4.3, $\operatorname{div} \mathscr{G}(E)=\operatorname{div} \mathcal{G}(E)-\operatorname{div} \mathbb{L}=0$ and hence $\operatorname{div} \mathbb{L}=\frac{1}{3} \varphi^{\prime}(e) \nabla e$. Without going into details (see, e.g., [Scala and Van Goethem 2016; Fosdick and Royer-Carfagni 2004]), [L then plays the role of a constraint reaction to ensure the condition $\operatorname{div} \mathscr{G}(E)=0$, and one could take $\mathbb{L}$ of the form $\mathbb{L}=\mathbb{C} \nabla^{S} w$ for a certain elasticity kind of tensor $\mathbb{C}$ and $w$ an associated vector field. 
Furthermore, one has $(\operatorname{Curl} \mathcal{G}(E))_{i j}=\beta(\operatorname{Curl} E)_{i j}-\frac{1}{3} \epsilon_{i j k} \varphi^{\prime}(\operatorname{tr} E) \partial_{k} \operatorname{tr} E$. It follows that

$$
\operatorname{Curl} \mathcal{G}(E)\left|\operatorname{Curl}^{t} E=\beta \operatorname{Curl} E\right| \operatorname{Curl}^{t} E+\varphi^{\prime}(\operatorname{tr} E) \mid(\nabla \operatorname{tr} E)^{2} .
$$

5.2. Energy estimates. For simplicity, the estimates will be done taking $\alpha=1$.

Theorem 17. Under Assumption 15, let $E$ be a solution of (5-1). Then

$$
\frac{d}{d t}\|E(t)\|_{L^{2}}^{2} \leq C\|E(t)\|_{L^{2}}^{2}
$$

for some $C>0$. Moreover,

$$
\|E\|_{L^{\infty}\left(0, T ; L^{2}\right)}+\|E\|_{L^{2}\left(0, T ; H^{2}\right)}^{2}+\left\|\frac{d E}{d t}\right\|_{L^{2}\left(0, T ; H^{-2}\right)} \leq C\left\|E_{0}\right\|_{L^{2}}^{2} .
$$

These estimates also hold for $E$ a solution of (5-4).

Proof. By (5-6), the polynomial $\sum_{i=1}^{2 p-2} \rho_{i} v^{i}$ is bounded from below by a constant. Hence, by (5-7), there exists $\tilde{c} \geq 0$ such that

$$
\operatorname{Curl} \mathcal{G}(E(t)) \mid \operatorname{Curl}^{t} E(t) \geq-\beta\|\operatorname{Curl} E(t)\|_{L^{2}}^{2}-\tilde{c}\|\nabla \operatorname{tr} E(t)\|_{L^{2}}^{2} .
$$

Denoting $\mathcal{C}(E(t)):=\beta\|\operatorname{Curl} E(t)\|_{L^{2}}^{2}+\tilde{c}\|\nabla \operatorname{tr} E(t)\|_{L^{2}}^{2} \geq 0$ and letting $F=E$ in (5-2), one has

$$
\begin{aligned}
& \frac{d}{d t} \frac{1}{2}\|E(t)\|_{L^{2}}^{2}+\mathbb{M} \text { inc } E(t)|\operatorname{inc} E-\mathcal{C}(E(t))-\mathbb{G}| E(t) \\
& \leq \frac{d}{d t} \frac{1}{2}\|E(t)\|_{L^{2}}^{2}+\mathbb{M} \operatorname{inc} E(t)|\operatorname{inc} E(t)+\operatorname{Curl} \mathcal{G}(E(t))| \operatorname{Curl}^{t} E(t)-\mathbb{G} \mid E(t)=0,
\end{aligned}
$$

and hence, there exists $C_{\mathbb{G}}>0$, a constant independent of $E$, such that

$$
\begin{aligned}
\frac{d}{d t} \frac{1}{2}\|E(t)\|_{L^{2}}^{2}+\mathbb{M i n c} E(t) \mid & \operatorname{inc} E(t) \\
& \leq \mathcal{C}(E(t))+\mathbb{G} \mid E(t) \leq C_{\mathbb{G}}\left(\|\nabla E(t)\|_{L^{2}}^{2}+\|E(t)\|_{L^{2}}\right) .
\end{aligned}
$$

The interpolation inequality and general Cauchy inequality [Evans 2010] yield

$$
\begin{aligned}
\frac{d}{d t} \frac{1}{2}\|E(t)\|_{L^{2}}^{2}+\mathbb{M i n c} E(t) \mid \operatorname{inc} E(t) & \leq c\left(\|E(t)\|_{L^{2}}\|E(t)\|_{H^{2}}+\|E(t)\|_{L^{2}}\right) \\
& \leq c \varepsilon\|E(t)\|_{H^{2}}^{2}+\frac{4 \varepsilon+c}{4 \varepsilon}\|E(t)\|_{L^{2}}^{2}
\end{aligned}
$$

for some constant $c:=c_{\mathbb{G}}>0$, a constant independent of $E$. Furthermore, positivedefiniteness and coercivity (see Theorem 13) of $\mathbb{M}$ yield $C_{\mathbb{M}}\|E\|_{H^{2}} \leq \mathbb{M}$ inc $E \mid$ inc $E$ 
for some constant $C_{\mathbb{M}}>0$. Thus, it follows from Theorem 13 and by choosing $\varepsilon$ small enough that

$$
\frac{d}{d t} \frac{1}{2}\|E(t)\|_{L^{2}}^{2} \leq \frac{d}{d t} \frac{1}{2}\|E(t)\|_{L^{2}}^{2}+\bar{C}\|E(t)\|_{H^{2}}^{2} \leq \frac{4 \varepsilon+c}{2 \varepsilon} \frac{1}{2}\|E(t)\|_{L^{2}}^{2}
$$

for some $\bar{C}(t) \geq 0$ (in the sequel, the dependencies of the constants on $\mathbb{G}$ and $\mathbb{M}$ are omitted for conciseness). As a consequence of the differential form of the Gronwall lemma [Evans 2010, §B.2.j] and (5-11), we deduce that

$$
\max _{t \in[0, T]}\|E(t)\|_{L^{2}} \leq C\left\|E_{0}\right\|_{L^{2}}
$$

for some constant $C>0$. Moreover, by (5-10) and time integration in $[0, T]$,

$$
\int_{0}^{t} \frac{d}{d s} \frac{1}{2}\|E(s)\|_{L^{2}}^{2} d s+\bar{C} \int_{0}^{t}\|E(s)\|_{H^{2}}^{2} d s \leq \widehat{C} \int_{0}^{t}\|E(s)\|_{L^{2}}^{2} d s
$$

for some $\widehat{C} \geq 0$. Hence, by (5-12),

$$
\frac{1}{2}\|E(t)\|_{L^{2}}^{2}-\frac{1}{2}\|E(0)\|_{L^{2}}^{2}+\bar{C} \int_{0}^{t}\|E(s)\|_{H^{2}}^{2} d s \leq C \widehat{C} T\left\|E_{0}\right\|_{L^{2}},
$$

and thus,

$$
\|E\|_{L^{2}\left(0, T ; H^{2}\right)}^{2}:=\int_{0}^{T}\|E(t)\|_{H^{2}}^{2} d t \leq \frac{2 \widehat{C} C T+1}{2 \bar{C}}\left\|E_{0}\right\|_{L^{2}}^{2} .
$$

To conclude, take any $V \in H_{0}^{2}\left(\Omega ; \mathbb{M}^{3}\right)$ and let $F=V$ in (5-2).

Set $V=V^{S}+V^{A}$, the symmetric-skewsymmetric decomposition of $V$, and $V^{S}=\nabla^{S} v+V^{0}$, the Beltrami decomposition of its symmetric part, with $V^{0} \in$ $\mathcal{H}_{0}(\Omega)$. Then, by means of some integration by parts,

$$
\begin{aligned}
\frac{d E}{d t}|V+\mathbb{M i n c} E| \text { inc } V & +\mathcal{G}(E) \mid \text { inc } V-\mathbb{G} \mid V \\
& =\frac{d E}{d t} \mid V^{0}+M \text { inc } E \mid \text { inc } V^{0}+\mathcal{G}(E) \mid \text { inc } V^{0}-\mathbb{G} \mid V^{0} \\
& =0 .
\end{aligned}
$$

Thus, we have for some constants $C_{1}, C_{2}^{i}>0$

$$
\left|\frac{d E}{d t}\right| V \mid \leq C_{1}\|E\|_{H^{2}}\|V\|_{H^{2}}+\sum_{i=0}^{2 p-1} C_{2}^{i}\|E\|_{L^{2}}^{i}\|V\|_{H^{2}},
$$

and hence, by (5-12) and (5-15) and with a nonrelabeled constant $C>0$,

$$
\left\|\frac{d E}{d t}\right\|_{L^{2}\left(0, T ; H^{-2}\right)}:=\int_{0}^{T}\left\|\frac{d E}{d t}\right\|_{H^{-2}} d t \leq C\left(\left\|E_{0}\right\|_{L^{2}}^{2}+\sum_{i=0}^{2 p-1}\left\|E_{0}\right\|_{L^{2}}^{i}\right),
$$


where $H^{-2}(\Omega):=\left(H_{0}^{2}\left(\Omega ; \mathbb{M}^{3}\right)\right)^{\prime}$. The proof follows from (5-12), (5-14), and (5-16) since, for the second statement, it suffices to take $\varphi \equiv 0$.

5.3. Existence and uniqueness of the weak solution. It is now well-known that the energy estimates of Theorem 17 and classical decomposition in discrete subspaces of $H^{2}$, the so-called Galerkin approximation (see, e.g., [Evans 2010; Temam 1997; Roubíček 2005]), yield the following theorem. Note that compactness is recovered in $H^{2}\left(\Omega ; \mathbb{S}^{3}\right)$ while the divergence-free properties also pass to the limit. Therefore, the solution belongs to $\mathcal{H}_{0}(\Omega)$ by the second statement of Lemma 8 .

Theorem 18. There exists a unique weak solution $E$ of (5-1) and (5-4) in $\mathcal{H}_{0}(\Omega)$. Moreover, $E \in \mathscr{C}\left(0, T ; H^{-2}\right)$.

Note that continuity in time is an immediate consequence of (5-9).

\section{Discussion}

6.1. Tensor version of Cahn-Hilliard. The derived equations are similar in form to the well-known Cahn-Hilliard equations, but here the variable is a divergencefree tensor $E$. Recall the strong form of (5-1) in $\Omega$ :

$$
\alpha \frac{d E}{d t}(t)+\operatorname{inc}(\mathbb{M} \text { inc } E(t)+\mathscr{G}(E(t)))=0 .
$$

Recall the identity $\operatorname{tr} \operatorname{inc} A=\Delta \operatorname{tr} A-\operatorname{div} \operatorname{div} A$. Then Assumption 15 yields

$$
\operatorname{trinc} \mathscr{G}(E)=\Delta \operatorname{tr}(\mathcal{G}(E)-\mathbb{L})=-\Delta \operatorname{tr} \mathbb{L}+\beta \operatorname{tr} E-\varphi(\operatorname{tr} E)
$$

since $\operatorname{tr}$ inc $A=\Delta \operatorname{tr} A$ for solenoidal fields $A$. Assume also that $\mathbb{M}=2 \tilde{\mu} \rrbracket_{4}+\tilde{\lambda} \rrbracket_{2} \otimes \mathbb{q}_{2}$ for some $\tilde{\mu}>0$, and set $\tilde{\beta}:=2(\tilde{\mu}+\tilde{\lambda})$.

Let us introduce

$$
e:=\operatorname{tr} E
$$

and compute the trace of (6-1). By (6-2),

$$
\begin{aligned}
\alpha e^{\prime}(t) & =\operatorname{trinc}(-\mathbb{M} \operatorname{inc} E(t)-\mathscr{G}(E(t))) \\
& =\Delta(-\tilde{\beta} \Delta e(t)-\beta e(t)+\varphi(e(t))+\operatorname{tr} \mathbb{L}),
\end{aligned}
$$

or more simply,

$$
\alpha e^{\prime}(t)=\Delta(-\tilde{\beta} \Delta e(t)+\psi(e(t))), \quad \psi(e):=\varphi(e)-\beta e+\operatorname{tr} \mathbb{L},
$$

which is recognized as the classical scalar version of the Cahn-Hilliard equation for $e$ with the nonlinear term $\psi$. Note that, in the classical derivation of the CahnHilliard equation, $\tilde{\beta}$ should depend on a small parameter related to a scaling in the free energy. In terms of our model, the part of the strain that is relevant for the variations of dislocation density, i.e., $E=\epsilon^{0}$ (by the relation $\operatorname{Curl} \kappa=\operatorname{inc} \epsilon^{0}$ ), 
has a trace $e$ and therefore is interpreted as dislocation-induced variation of matter density. It is remarkable that $e$ obeys the law (6-4).

Regarding its boundary conditions, it is already known by (3-8) that $e=0$ on $\partial \Omega$. We also assume that $\Delta e=0$ on $\partial \Omega$ and the initial condition $e(0)=\operatorname{tr} E_{0}$. It is wellknown that (6-3) is well-posed (see [Elliott and Stuart 1996] for this particular choice of boundary conditions) though the solution might only be unique up to some gauges since $\partial_{N} e$ is not fixed.

Moreover, $\frac{d}{d t} \int_{\Omega} e d x=-\int_{\partial \Omega} \tilde{\beta} \partial_{N} \Delta e d S(x)+\int_{\partial \Omega}\left(\varphi^{\prime}(e)-\beta\right) \partial_{N} e d S(x)$ clearly follows. From a physical viewpoint, this property simply reflects the inflow of point defects. In fact, any variation of $e$ is due to the change in interstitial and vacancy densities. In some sense, $e$ might be viewed as a point-defect density: positive in the case of an excess of interstitials and negative if vacancies exceed interstitials. Furthermore, assuming that $e$ depends on the temperature $T$, one has a leading boundary inflow proportional to the normal temperature gradient, i.e., given by $\left(\varphi^{\prime}(e)-\beta\right) e^{\prime}(T) \partial_{N} T$. Hence, the point defects will be conserved: $\frac{d}{d t} \int_{\Omega} e d x=0$ as soon as the normal temperature gradient vanishes at the boundary. Otherwise, point defects will be introduced or removed from the boundary. Furthermore, the fact that $e=0$ on $\partial \Omega$ means that point defects are only present inside $\Omega$. Note that point defects on the boundary is somewhat nonsensical since an excess/lack of atoms indeed changes the boundary location. Recall also that dislocations are nucleated by the collapse of point-defect clusters. Hence, determining their density is crucial for dislocation modeling.

Note also that $e$ is the potential yielding the bulk dislocation force $\nabla e$ in (3-3). Therefore, the work done by this force only depends on the variation of point-defect density at the path endpoints. Specifically, the displacement is a solution to

$$
\left\{\begin{aligned}
\rho \partial_{t}^{2} u-\operatorname{div}\left(\mathbb{A} \nabla^{S} u\right) & =f+\lambda \nabla e & & \text { in } \Omega \times[0, T], \\
\left(\mathbb{A} \nabla^{S} u\right) N & =g-\lambda e N & & \text { on } \partial \Omega \times[0, T]
\end{aligned}\right.
$$

as coupled with the point-defect density

$$
\left\{\begin{array}{rlrl}
\partial_{t} e(t)+\Delta(\tilde{\beta} \Delta e(t)+\psi(e(t))) & =0 & & \text { in } \Omega \times[0, T], \\
e=\Delta e=0 & & \text { on } \partial \Omega \times[0, T], \\
e(0) & =e_{0} & & \text { in } \Omega \times[0, T],
\end{array}\right.
$$

where the bulk force term in the right-hand side will be explained in the next subsection. It represents a dissipative force related to point defects as a source or sink.

6.2. Comment about the forcing term. Note that tr $\mathbb{L}$ in (6-3) stands for an external time-dependent field in Gurtin's formalism of microforce balance [1996].

Let us rewrite (4-10) as $\delta_{\epsilon^{0}} \psi_{\text {dislo }}=\mathbb{P}^{C}+\mathbb{P}^{D}$, where the symmetric gradient $\mathbb{P}^{C}$ is impactless on the mechanical dissipation. Accordingly, let $\psi_{\text {dislo }}=\psi_{\text {dislo }}^{C}+\psi_{\text {dislo }}^{D}$, where the first term is a conservative contribution while the latter is dissipative. 
One has $\operatorname{div} \mathbb{P}^{D}=\operatorname{div} \delta_{\epsilon^{0}} \psi_{\text {dislo }}^{D}=\delta_{\epsilon^{0}}^{2} \psi_{\text {dislo }}^{D} \nabla \epsilon^{0}=0$, which implies that $\psi_{\text {dislo }}^{D}$ must be affine in $\epsilon^{0}$. Hence,

$$
\psi_{\text {dislo }}^{D}\left(\epsilon^{0}\right)=\operatorname{inc} \mathbb{K}_{0} \cdot \epsilon^{0}+\mathbb{C}_{0}
$$

for some constant tensor $\mathbb{C}_{0}$. Now, from the expression of the dissipation term of (4-11), inc $\mathbb{K}_{0}\left|\dot{\epsilon}^{0}=\mathbb{K}_{0}\right|$ inc $\dot{\epsilon}^{0}=\mathbb{K}_{0} \mid$ Curl $\dot{\kappa}$, one recognizes $\mathbb{K}_{0}$ as a thermodynamic force.

Now, letting

$$
\psi_{\text {dislo }}^{C}\left(\epsilon^{0}\right)=\psi^{C}\left(\operatorname{tr} \epsilon^{0}\right)+\tilde{\psi}^{C}\left(\nabla \operatorname{tr} \epsilon^{0}\right),
$$

with $\psi^{C}(e)=\int_{0}^{e} \varphi(v) d v-\frac{1}{2} \beta e^{2}+\operatorname{tr} \llbracket e$ and $\tilde{\psi}^{C}(\nabla e)=\frac{1}{2} \tilde{\beta} \nabla e \cdot \nabla e,(6-6)$ can be rewritten as the classical parabolic diffusion equation of the form

$$
\left\{\begin{aligned}
\partial_{t} e(t)+\operatorname{div} j=0 & \text { in } \Omega \times[0, T] \text { with } j:=-\nabla \mu \\
& \text { and } \mu:=\delta_{e}\left(\psi^{C}(e)+\tilde{\psi}^{C}(\nabla e)\right), \\
e=\Delta e=0 & \text { on } \partial \Omega \times[0, T], \\
e(0)=e_{0} & \text { in } \Omega \times[0, T] .
\end{aligned}\right.
$$

6.3. Gradient flow. Let us assume the existence of a scalar $\mathscr{H}$ such that

$$
\text { (inc } \left.\mathscr{G}\left(\epsilon^{0}\right), F\right)=\lim _{\epsilon \rightarrow 0} \frac{\mathscr{H}\left(\epsilon^{0}+\epsilon F\right)-\mathscr{H}\left(\epsilon^{0}\right)}{\epsilon}
$$

for every $F \in \mathcal{H}_{0}(\Omega)$. Note that, for a nonlinear term of the form (5-5), one has $\mathscr{H}\left(\epsilon^{0}\right)=-\left(\mathbb{G}, \epsilon^{0}\right)+\frac{1}{2} \beta\left(\operatorname{Curl}^{t} \epsilon^{0}, \operatorname{Curl} \epsilon^{0}\right)-\frac{1}{3} \phi\left(\operatorname{tr} \epsilon^{0}\right) \rrbracket_{2}$, where $\varphi=\phi^{\prime}$. By defining the incompatibility energy as

$$
\mathscr{E}\left(\epsilon^{0}\right):=\int_{\Omega} \frac{1}{2} \mathbb{M} \text { inc } \epsilon^{0} \cdot \operatorname{inc} \epsilon^{0} d x+\mathscr{H}\left(\epsilon^{0}\right),
$$

it is obvious by integration by parts that the Gâteaux derivative of $\mathscr{E}$ at $\epsilon^{0} \in \mathscr{C}^{\infty}(\Omega)$ in the direction $F \in \mathcal{H}_{0}(\Omega)$ reads $\left\langle\operatorname{grad}_{L^{2}}^{\mathcal{H}_{0}} \mathscr{E}\left(\epsilon^{0}\right), F\right\rangle=\int_{\Omega} \operatorname{inc}\left(\mathbb{M}\right.$ inc $\left.\epsilon^{0}+\mathscr{G}\left(\epsilon^{0}\right)\right) \cdot F d x=\int_{\Omega}\left(\mathbb{M}\right.$ inc $\left.\epsilon^{0}+\mathscr{G}\left(\epsilon^{0}\right)\right) \cdot \operatorname{inc} F d x$. Thus, for a general $\epsilon^{0} \in \mathcal{H}(\Omega)$, one defines the $\mathcal{H}^{-1}$-gradient

$$
\left\langle\operatorname{grad}_{\mathcal{H}^{-1}}^{\mathcal{H}_{0}} \mathscr{E}\left(\epsilon^{0}\right), F\right\rangle:=\int_{\Omega}\left(\mathbb{M} \text { inc } \epsilon^{0}+\mathscr{G}\left(\epsilon^{0}\right)\right) \cdot \operatorname{inc} F d x,
$$

where $\mathcal{H}^{-1}:=\left(\mathcal{H}_{0}(\Omega)\right)^{\prime}$. Due to the Riesz theorem, the linear and continuous map $\operatorname{grad}_{\mathcal{H}^{-1}}^{\mathcal{H}_{0}} \mathscr{E}\left(\epsilon^{0}\right)$ is associated with a unique $F^{0} \in \mathcal{H}_{0}(\Omega)$ such that inc inc $F^{0}=$ $\operatorname{inc}\left(\mathbb{M}\right.$ inc $\left.\epsilon^{0}+\mathscr{G}\left(\epsilon^{0}\right)\right)$, so $\left\langle\operatorname{grad}_{\mathcal{H}^{-1}}^{\mathcal{H}_{0}} \mathscr{E}\left(\epsilon^{0}\right), F\right\rangle:=\int_{\Omega}$ inc $F^{0}$. inc $F d x$. By analogy with the scalar Cahn-Hilliard system, let us call - inc $F^{0}$ the "chemical potential". 
Furthermore, $\left\|\operatorname{grad}_{\mathcal{H}^{-1}}^{\mathcal{H}_{0}} \mathscr{E}\left(\epsilon^{0}\right)\right\|_{\mathcal{H}^{-1}}^{2}=\|$ inc $F^{0} \|_{L^{2}}^{2}=\left(\mathbb{M} \text { inc } \epsilon^{0}+\mathscr{G}\left(\epsilon^{0}\right) \text {, inc } F^{0}\right)_{L^{2}}$, and hence, our model evolution equation can be written as the $\mathcal{H}^{-1}$-gradient flow

$$
\partial_{t} \epsilon^{0}=-\frac{1}{\alpha} \operatorname{grad}_{\mathcal{H}^{-1}}^{\mathcal{H}_{0}} \mathscr{E}:=-\frac{1}{\alpha} \operatorname{inc}\left(\mathbb{M} \operatorname{inc} \epsilon^{0}+\mathscr{G}\left(\epsilon^{0}\right)\right) .
$$

Now, (6-8) implies that

$$
\begin{array}{r}
\frac{d}{d t} \mathscr{E}=\left\langle\operatorname{grad}_{\mathcal{H}^{-1}}^{\mathcal{H}_{0}} \mathscr{E}, \dot{\epsilon}^{0}\right\rangle=\left\langle\operatorname{inc}\left(\mathbb{M i n c} \epsilon^{0}+\mathscr{G}\left(\epsilon^{0}\right)\right), \dot{\epsilon}^{0}\right\rangle=\left(\mathbb{M} \text { inc } \epsilon^{0}+\mathscr{G}\left(\epsilon^{0}\right), \operatorname{inc} \dot{\epsilon}^{0}\right)_{L^{2}} \\
=-\frac{1}{2}\left(\alpha\left|\dot{\epsilon}^{0}\right|^{2}+\frac{1}{\alpha}\left\|\operatorname{grad}_{\mathcal{H}^{-1}}^{\mathcal{H}_{0}} \mathscr{E}\right\|_{\mathcal{H}^{-1}(\Omega)}^{2}\right)=-\frac{1}{\alpha} \| \operatorname{grad}_{\mathcal{H}^{-1}}^{\mathcal{H}_{0}} \mathscr{E}_{\mathcal{H}^{-1}(\Omega)}^{2} \leq 0,
\end{array}
$$

and hence, (4-16) can be written as

$$
0 \leq \mathscr{D}=-\frac{d}{d t}\left(\mathscr{E}_{\beta}\left(\epsilon^{0}, \kappa\right)+\mathscr{E}\left(\epsilon^{0}\right)\right)=\mathscr{D}_{\text {dislo }}+\mathscr{D}_{\text {incomp }}
$$

with the dislocation-induced dissipation term $\mathscr{D}_{\text {dislo }}:=-\frac{d}{d t} \mathscr{E}_{\beta}\left(\epsilon^{0}, \kappa\right)$ vanishing as soon as the free energy is independent of $\kappa$ while the incompatibility-induced dissipation term $\mathscr{D}_{\text {incomp }}:=(1 / \alpha)\left\|\operatorname{grad}_{\mathcal{H}^{-1}}^{\mathcal{H}_{0}} \mathscr{E}\right\|_{\mathcal{H}^{-1}(\Omega)}^{2}=-\frac{d}{d t} \mathscr{E}$ is due to the dependence of the free energy on $\operatorname{Curl} \kappa=$ inc $\epsilon^{0}$. Thus, the incompatibility-induced dissipated energy in $[0, T]$ is decreasing since

$$
\mathscr{E}_{\text {incomp }}:=\int_{0}^{T} \mathscr{D}_{\text {incomp }} d t=\mathscr{E}(0)-\mathscr{E}(T) \geq 0,
$$

and hence, stationarity means that minimization is reached. Therefore, the system should progress toward a stable equilibrium state, a global minimizer of $\mathscr{E}$. This is the basic justification for minimization schemes in a quasistatic setting. Note that maximizing the incompatibility-induced dissipated energy is equivalent to minimizing the incompatibility energy $\mathscr{E}(T)$. Set $\beta=\phi=0$. Then for long-time behavior, one can simply consider the variational problem

$$
\inf _{E \in \mathcal{H}^{\star} \subset \mathcal{H}(\Omega)} \int_{\Omega}\left(\frac{1}{2} \mathbb{M} \text { inc } E \cdot \operatorname{inc} E-\mathbb{G} \cdot E\right) d x
$$

as done in [Amstutz and Van Goethem 2016].

6.4. Concluding remark. This work represents the first step towards a deep understanding of time evolution of dislocation networks at the mesoscale. Its principal aim was to shed light on the importance of the incompatibility operator in the study of dislocations and to propose an evolution in time of the dislocation-induced strain. This required first introducing and/or recalling some properties of this operator as well as its appropriate functional space. The evolution law is based on thermodynamical principles and on the postulate of maximal dissipation adopted for the model internal variables. It turns out that, as a consequence of the second law 
of thermodynamics, the evolution takes the form of the tensor formulation of the Cahn-Hilliard system

$$
\alpha \partial_{t} \epsilon^{0}=\text { inc } \mu,
$$

where $\mu:=-\left(\mathbb{M}\right.$ inc $\left.\epsilon^{0}+\mathscr{G}\left(\epsilon^{0}\right)\right)$ is called the tensor "chemical potential" with $\mathbb{M}$ a positive-definite fourth-rank tensor with the dimensions of a force times a squared distance.

Moreover, the classical scalar Cahn-Hilliard system is recovered for the trace of the dislocation strain, called $e$, which is interpreted as the density of point defects since it allows one to change the solid density by adding or removing single atoms. Remark that this fourth-order equation for $e$ is not classical at all since point defects are classically modeled by second-order reaction-diffusion equations [Van Goethem et al. 2008]. Furthermore, $\nabla e$ also appears to play the role of a conservative bulk force in the displacement equation. Note also that the thermodynamic derivation of the model equations leads to a nonlinear term whose explicit expression is not known. For simplicity, we have considered a general polynomial term in the trace of $\epsilon^{0}$, i.e., in $e$. Of course, more elaborate choices can be made with a view of a general model, but note that the physical sense of the other two invariants of the dislocation strain is not clear.

A crucial quantity we have introduced is the incompatibility energy

$$
\mathscr{E}(E):=\int_{\Omega}\left(\frac{1}{2} \mathbb{M} \text { inc } E \cdot \operatorname{inc} E+\frac{1}{2} \beta \text { inc } E \cdot E-\frac{1}{3} \phi(\operatorname{tr} E)-\mathbb{G} \cdot E\right) d x
$$

with $\beta>0$ a scalar with the dimensions of a force and the scalar $\phi$ and solenoidal tensor $\mathbb{G}$ with the dimensions of a surface force density. Furthermore, $\phi$ is a nonlinear potential depending on $e=\operatorname{tr} E$ and related to point defects. We have shown that the time evolution of the incompatible strain $\epsilon^{0}$ is given by (6-9), which in turn yields the energy equation

$$
\alpha \frac{d}{d t} \mathscr{E}\left(\epsilon^{0}\right)+\left\|\operatorname{grad}_{\mathcal{H}^{-1}}^{\mathcal{H}_{0}} \mathscr{E}\right\|_{\mathcal{H}^{-1}(\Omega)}^{2}=0
$$

for some $\alpha>0$. Therefore, solutions for large times, $\epsilon_{\infty}^{0}$, should approach the minima of the incompatibility energy, namely

$$
\mathscr{E}\left(\epsilon_{\infty}^{0}\right)=\inf _{E \in \mathcal{H}^{\star} \subset \mathcal{H}(\Omega)} \mathscr{E}(E),
$$

which may therefore be considered the associated quasistatic variational problem.

This work and the formalism introduced are expected to clear the way for more involved, complete, and realistic models for the evolution of dislocation networks at the mesoscale. In particular, the interface dynamics in a model of point defects and dislocations in single crystals will be discussed in future work. 


\section{Acknowledgements}

The author was supported by the FCT Starting Grant "Mathematical theory of dislocations: geometry, analysis, and modeling" (IF/00734/2013). The author thanks Samuel Amstutz for fruitful discussions about incompatibility-based models.

\section{References}

[Agmon et al. 1964] S. Agmon, A. Douglis, and L. Nirenberg, "Estimates near the boundary for solutions of elliptic partial differential equations satisfying general boundary conditions, II", Comm. Pure Appl. Math. 17 (1964), 35-92.

[Amstutz and Van Goethem 2016] S. Amstutz and N. Van Goethem, "Analysis of the incompatibility operator and application in intrinsic elasticity with dislocations", SIAM J. Math. Anal. 48:1 (2016), 320-348.

[Berdichevsky 2006] V. L. Berdichevsky, "Continuum theory of dislocations revisited", Contin. Mech. Thermodyn. 18:3-4 (2006), 195-222.

[Bolik and von Wahl 1997] J. Bolik and W. von Wahl, "Estimating $\nabla \boldsymbol{u}$ in terms of div $\boldsymbol{u}, \operatorname{curl} \boldsymbol{u}$, either $(v, \boldsymbol{u})$ or $v \times \boldsymbol{u}$ and the topology", Math. Methods Appl. Sci. 20:9 (1997), 737-744.

[Elliott and Stuart 1996] C. M. Elliott and A. M. Stuart, "Viscous Cahn-Hilliard equation, II: Analysis”, J. Differential Equations 128:2 (1996), 387-414.

[Evans 2010] L. C. Evans, Partial differential equations, 2nd ed., Graduate Studies in Mathematics 19, American Mathematical Society, Providence, RI, 2010.

[Fosdick and Royer-Carfagni 2004] R. Fosdick and G. Royer-Carfagni, "Stress as a constraint reaction in rigid bodies”, J. Elasticity 74:3 (2004), 265-276.

[Gurtin 1996] M. E. Gurtin, "Generalized Ginzburg-Landau and Cahn-Hilliard equations based on a microforce balance”, Phys. D 92:3-4 (1996), 178-192.

[Henrot and Pierre 2005] A. Henrot and M. Pierre, Variation et optimisation de formes: une analyse géométrique, Mathématiques \& Applications 48, Springer, Berlin, 2005.

[Kozono and Yanagisawa 2009] H. Kozono and T. Yanagisawa, " $L^{r}$-variational inequality for vector fields and the Helmholtz-Weyl decomposition in bounded domains", Indiana Univ. Math. J. 58:4 (2009), 1853-1920.

[Lemaitre and Chaboche 1988] J. Lemaitre and J.-L. Chaboche, Mécanique des matériaux solides, 2nd ed., Dunod, Paris, 1988.

[Maggiani et al. 2015] G. B. Maggiani, R. Scala, and N. Van Goethem, "A compatible-incompatible decomposition of symmetric tensors in $L^{p}$ with application to elasticity", Math. Methods Appl. Sci. 38:18 (2015), 5217-5230.

[Maugin 2015] G. A. Maugin, "The saga of internal variables of state in continuum thermo-mechanics (1893-2013)", Mech. Res. Commun. 69 (2015), 79-86.

[Ottosen and Ristinmaa 2005] N. S. Ottosen and M. Ristinmaa, The mechanics of constitutive modeling, Elsevier, Amsterdam, 2005.

[Roubíček 2005] T. Roubíček, Nonlinear partial differential equations with applications, International Series of Numerical Mathematics 153, Birkhäuser, Basel, 2005.

[Scala and Van Goethem 2016] R. Scala and N. Van Goethem, "Constraint reaction and the PeachKoehler force for dislocation networks", preprint, 2016, Available at https://hal.archives-ouvertes.fr/ hal-01213861v2. To appear in Math. Mech. Complex Sys. 
[Temam 1997] R. Temam, Infinite-dimensional dynamical systems in mechanics and physics, 2nd ed., Applied Mathematical Sciences 68, Springer, New York, 1997.

[Van Goethem 2014] N. Van Goethem, "Thermodynamic forces in single crystals with dislocations", Z. Angew. Math. Phys. 65:3 (2014), 549-586.

[Van Goethem 2015] N. Van Goethem, "Cauchy elasticity with dislocations in the small strain assumption”, Appl. Math. Lett. 46 (2015), 94-99.

[Van Goethem 2016a] N. Van Goethem, "Direct expression of incompatibility in curvilinear systems", ANZIAM J. 58:1 (2016), 33-50.

[Van Goethem 2016b] N. Van Goethem, "Incompatibility-governed singularities in linear elasticity with dislocations", Math. Mech. Solids (online publication May 2016).

[Van Goethem et al. 2008] N. Van Goethem, A. de Potter, N. Van den Bogaert, and F. Dupret, "Dynamic prediction of point defects in Czochralski silicon growth: an attempt to reconcile experimental defect diffusion coefficients with the $V / G$ criterion", J. Phys. Chem. Solids 69:2-3 (2008), $320-324$.

[von Wahl 1992] W. von Wahl, "Estimating $\nabla u$ by div $u$ and curl $u$ ", Math. Methods Appl. Sci. 15:2 (1992), 123-143.

Received 25 Jan 2016. Revised 23 May 2016. Accepted 30 Jun 2016.

Nicolas VAn Goethem: vangoeth@fc.ul.pt

Departamento de Matemática, CMAF CIO, Universidade de Lisboa, Alameda da Universidade, C6, 1749-016 Lisboa, Portugal 
EDITORIAL BOARD

ANTONIO CARCATERRA

ERIC A. CARLEN

FRANCESCO DELL'ISOLA

RAFFAELE ESPOSITO

ALBERT FANNJIANG

Gilles A. FranCFORT

Pierangelo MARCATI

JEAN-JACQUES MARIGO

PETER A. MARKOWICH

MARTIN OSTOJA-STARZEWSKI

PIERRE SEPPECHER

DAVID J. STEIGMANN

PAUl STEINMANN

PierRe M. SuQueT

MANAGING EDITORS

MICOL AMAR

CORRADO LATTANZIO

ANGELA MADEO

MARTIN OSTOJA-STARZEWSKI

ADVISORY BOARD

ADNAN AKAY

Holm AltenBaCH

MICOL AMAR

HARM ASKES

TEODOR ATANACKOVIĆ

VICTOR BERDICHEVSKY

GUY BOUCHITTÉ

ANDREA BRAIDES

ROBERTO CAMASSA

MAURO CARFORE

ERIC DARVE

FELIX DARVE

ANNA DE MASI

GianPiEtro DEL Piero

EMMANUELE Di BENEDETTO

BERNOLD FIEDLER

IRENE M. GAMBA

DAVID Y. GAO

SERGEY GAVRILYUK

TIMOTHY J. HEALEY

DOMINIQUE JEULIN

ROGER E. KHAYAT

CORRADO LATTANZIO

ROBERT P. LIPTON

ANGELO LUONGO

ANGELA MADEO

JUAN J. MANFREDI

CARLO MARCHIORO

GÉRARD A. MAUGIN

ROBERTO NATALINI PATRIZIO NEFF

ANDREY PIATNITSKI

ERRICO PRESUTTI

MARIO PULVIRENTI

LUCIO RUSSO

Miguel A. F. SANJUAN

PATRICK SElVADURAI

ALEXANDER P. SEYRANIAN

MIROSLAV ŠILHAVÝ

GUIDO SWEERS

ANTOINETTE TORDESILLAS

LEV TRUSKINOVSKY

JUAN J. L. VELÁZQUEZ VINCENZO VESPRI ANGELO VULPIANI msp.org/memocs

Università di Roma "La Sapienza", Italia

Rutgers University, USA

(CO-CHAIR) Università di Roma "La Sapienza", Italia

(TREASURER) Università dell'Aquila, Italia

University of California at Davis, USA

(CO-CHAIR) Université Paris-Nord, France

Università dell'Aquila, Italy

École Polytechnique, France

DAMTP Cambridge, UK, and University of Vienna, Austria

(CHAIR MANAGING EDITOR) Univ. of Illinois at Urbana-Champaign, USA

Université du Sud Toulon-Var, France

University of California at Berkeley, USA

Universität Erlangen-Nürnberg, Germany

LMA CNRS Marseille, France

Università di Roma "La Sapienza", Italia

Università dell'Aquila, Italy

Université de Lyon-INSA (Institut National des Sciences Appliquées), France

(CHAIR MANAGING EDITOR) Univ. of Illinois at Urbana-Champaign, USA

Carnegie Mellon University, USA, and Bilkent University, Turkey

Otto-von-Guericke-Universität Magdeburg, Germany

Università di Roma "La Sapienza", Italia

University of Sheffield, UK

University of Novi Sad, Serbia

Wayne State University, USA

Université du Sud Toulon-Var, France

Università di Roma Tor Vergata, Italia

University of North Carolina at Chapel Hill, USA

Università di Pavia, Italia

Stanford University, USA

Institut Polytechnique de Grenoble, France

Università dell'Aquila, Italia

Università di Ferrara and International Research Center MEMOCS, Italia

Vanderbilt University, USA

Freie Universität Berlin, Germany

University of Texas at Austin, USA

Federation University and Australian National University, Australia

Université Aix-Marseille, France

Cornell University, USA

École des Mines, France

University of Western Ontario, Canada

Università dell' Aquila, Italy

Louisiana State University, USA

Università dell'Aquila, Italia

Université de Lyon-INSA (Institut National des Sciences Appliquées), France University of Pittsburgh, USA

Università di Roma "La Sapienza”, Italia

Université Paris VI, France

Istituto per le Applicazioni del Calcolo "M. Picone", Italy

Universität Duisburg-Essen, Germany

Narvik University College, Norway, Russia

Università di Roma Tor Vergata, Italy

Università di Roma "La Sapienza”, Italia

Università di Roma “Tor Vergata”, Italia

Universidad Rey Juan Carlos, Madrid, Spain

McGill University, Canada

Moscow State Lomonosov University, Russia

Academy of Sciences of the Czech Republic

Universität zu Köln, Germany

University of Melbourne, Australia

École Polytechnique, France

Bonn University, Germany

Università di Firenze, Italia

Università di Roma La Sapienza, Italia

MEMOCS (ISSN 2325-3444 electronic, 2326-7186 printed) is a journal of the International Research Center for the Mathematics and Mechanics of Complex Systems at the Università dell'Aquila, Italy.

Cover image: "Tangle” by $\odot$ John Horigan; produced using the Context Free program (contextfreeart.org).

PUBLISHED BY

7 mathematical sciences publishers

nonprofit scientific publishing

http://msp.org/

(C) 2016 Mathematical Sciences Publishers 
Mathematics and Mechanics of Complex Systems vol. 4 no. 2

Constraint reaction and the Peach-Koehler force for dislocation networks

Riccardo Scala and Nicolas Van Goethem

Stability analysis of two coupled oscillators

Mickhail A. Guzev and Alexandr A. Dmitriev

Analysis of the electromagnetic reflection and transmission 153 through a stratified lossy medium of an elliptically

polarized plane wave

Fabio Mangini and Fabrizio Frezza

Dislocation-induced linear-elastic strain dynamics by a

Cahn-Hilliard-type equation

Nicolas Van Goethem

MEMOCS is a journal of the International Research Center for the Mathematics and Mechanics of Complex Systems at the Università dell' Aquila, Italy.

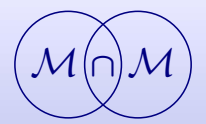

